DESY 94-164

September 1994

ESI 132 (1994)

hep-th/9409165

\title{
Characterizing invariants for local extensions of current algebras
}

\author{
KARL-HENNING REHREN \\ II. Institut für Theoretische Physik, Universität Hamburg, \\ D-22761 Hamburg (GERMANY) \\ Yassen S. Stanev and Ivan T. Todorov \\ Erwin Schrödinger International Institute for Mathematical Physics, \\ A-1090 Wien (Austria) \\ and \\ Institute for Nuclear Research and Nuclear Energy, \\ Bulgarian Academy of Sciences, BG-1784 Sofia (Bulgaria) *
}

\begin{abstract}
Pairs $\mathcal{A} \subset \mathcal{B}$ of local quantum field theories are studied, where $\mathcal{A}$ is a chiral conformal quantum field theory and $\mathcal{B}$ is a local extension, either chiral or two-dimensional. The local correlation functions of fields from $\mathcal{B}$ have an expansion with respect to $\mathcal{A}$ into conformal blocks, which are nonlocal in general. Two methods of computing characteristic invariant ratios of structure constants in these expansions are compared: $(a)$ by constructing the monodromy representation of the braid group in the space of solutions of the Knizhnik-Zamolodchikov differential equation, and (b) by an analysis of the local subfactors associated with the extension with methods from operator algebra (Jones theory) and algebraic quantum field theory. Both approaches apply also to the reverse problem: the characterization and (in principle) classification of local extensions of a given theory.
\end{abstract}

\section{Introduction}

The relevance of V. Jones' theory of (von Neumann) subfactors [1] for 2-dimensional (2D) models of critical behaviour was first recognized in the work of V. Pasquier on lattice models labelled by Dynkin diagrams [2]. A spectacular by-product of this realization was the ensuing $A D E$ classification of $s u(2)$ current algebra models and minimal conformal theories [3]. The above parallel was understood within the Haag-Kastler algebraic approach to local quantum field theory [4] in terms of the Doplicher-Haag-Roberts (DHR) theory of superselection sectors and particle statistics [5] applied to chiral algebras [6, 7], and provided an explanation for the Jones index as a measure for the violation of Haag duality (maximality of local observables) in a given representation, and relating it numerically to the statistical dimension [8].

In the cited work on subfactors in quantum field theory, the emphasis for the use of the theory of subfactors was its application to individual superselection sectors of a given

*permanent address 
local theory, and the derivation of invariant 'charge quantum numbers' such as statistical dimensions and Markov traces. In contrast, here we shall consider a pair of local theories, one extending the other, as a subfactor (actually, a net of local subfactors) and apply the properly adapted Jones theory to describe the 'position' of the subtheory in its extension. This point of view opens the way to a detailed understanding of the behaviour of superselection sectors when one passes from one theory to the other by a generalized Mackey induction and restriction prescription [9].

In particular, given that the position of a subtheory in another theory is encoded and characterized by a subfactor, then subfactor theoretical methods can be applied to conformal models and their local extensions, and must give detailed answers comparable with the $A D E$ classification and related results obtained by conventional methods of conformal quantum field theory.

The present article is a comparative study of conventional field theoretical methods on the one hand and the theory of subfactors on the other hand in application to the same problem: local extensions of local quantum field theories. A local extension is determined by the correlation functions of the extending fields. In chiral current algebra models of conformal field theory, the extending fields necessarily correspond to primary fields of the unextended theory with bosonic, i.e., integer conformal dimension $\Delta$. Their 4-point functions are linear (for chiral extensions) or bilinear (for 2D extensions) combinations of conformal block functions which are monodromy free inspite of the non-trivial braid group transformation of the individual conformal blocks. Moreover, unlike the chiral vertex operators of the unextended theory whose fusion rules coincide with the intrinsic composition law of superselection charges provided by the DHR theory, the extending local fields must satisfy truncated fusion rules which involve only other bosonic fields, and which are therefore only majorized by the DHR fusion.

Both the truncated fusion rules and the ratios of structure constants (amplitudes of conformal block functions) in the said combinations are characteristic quantities for a pair of a chiral current algebra and its extension. They are computed by both methods. In the first part of the article (Sects. 2 and 3), we study the monodromy behaviour of the solutions of the Knizhnik-Zamolodchikov (KZ) equation and compute the braid invariant quadratic forms which determine the local 4-point functions of the two-dimensional extensions. Apart from the generic two-dimensional extension (corresponding to the $A$ series of the $A D E$ classification), and the chiral $D$ series extensions which correspond to a global $\mathbb{Z}_{2}$ symmetry, we concentrate on the exceptional chiral $E_{6}$ and $E_{8}$ extensions of $s u(2)$ current algebras. We compute explicitly the relative amplitudes of the $A$ and $E$ theories, which turn out to be rational numbers. In the second part (Sects. 4 and 5), we study the position of the operator algebra of the unextended subtheory within its extension, in terms of the theory of subfactors. Remarkably, the relevant information already resides in a single pair of local von Neumann algebras. We analyze which quantities in the general theory of subfactors, when applied to a given local field extension, contain the desired information about the truncated fusion rules and the relevant ratios of structure constants. We describe how to compute these data in terms of the subtheory (interpreted as the physical observables) and its superselection structure.

While the first method will be easier to use in specific models and as long as one is interested only in 4-point functions, the second method is part of a general theory of local field extensions, confined neither to two dimensions nor to conformal quantum field theories. It covers also the standard situation of four-dimensional theories with a compact gauge group. (In this latter case, the method essentially reduces to harmonic analysis and partial wave expansions based on the representation theory and Clebsch-Gordan coefficients 
of the gauge group.) It has the advantage to treat all $n$-point functions at one stroke. However, in practice it requires to solve in a first step a complicated non-linear system for the 'generalized Clebsch-Gordan coefficients', which we have carried out only for the simplest model of a field extension which is not due to a gauge group.

The local extensions of chiral su(2) current algebras studied in Sects. 2 and 3 are distinguished to have the same stress-energy tensor as the original theory, the stress-energy tensor implicitly entering the analysis through the $\mathrm{KZ}$ equations. If the extending fields are currents of dimension $\Delta=1$, this means that the extension is a 'conformal embedding' [10]. On the other hand, in Sects. 4 and 5, we assume the index of the inclusion to be finite. Indeed, for pairs of chiral current algebras, these two selection criteria are equivalent. Namely, both the finiteness of the index and the triviality of the coset stress-energy tensor are equivalent to the finiteness of the branching of the vacuum representation of the extended theory upon restriction to the subtheory.

Let $\mathcal{A} \subset \mathcal{B}$ be a conformal embedding [10] of two chiral quantum field theories like the current algebras $\mathcal{A}_{10}\left(A_{1}\right) \subset \mathcal{A}_{1}\left(B_{2}\right)$ where $A_{1}=\operatorname{su}(2)$ and $B_{2}=\operatorname{spin}(5) \simeq \operatorname{sp}(4)$ refer to the Lie algebras underlying the current algebras, and the subscripts refer to the level 10 resp. 1 of the central extension. The embedding gives rise to a pair of braid-invariant quadratic forms $M$ and $\widetilde{M}$ in the space of 4-point conformal blocks of the subtheory $\mathcal{A}$ with four given external quantum numbers (superselection charges) such as isospins $I \leq k / 2$ for $\mathcal{A}=\mathcal{A}_{k}\left(A_{1}\right)$. The quadratic forms serve to express $2 \mathrm{D}$ correlation functions in terms of chiral conformal blocks, and turn out to completely characterize the model. The form $M$ corresponds to the 'diagonal' WZNW theory [11] over $\mathcal{A}$, i.e., to the $A_{k+1}$ theory in the $A D E$ classification of $s u(2)$ current algebra models at level $k[3]$. The eigenvalues $D_{\lambda}^{(k, I)}$ of $M$, in the case of 4 equal external isospins $I$, are the squares of the structure constants

$$
D_{\lambda}^{(k, I)} \equiv N_{I I \lambda}^{2} \quad\left(\lambda=0,1, \ldots \min (2 I, k-2 I) \equiv m_{k I}\right)
$$

for the $s$-channel fusion of two of the isospin $I$ charges into isospin $\lambda$ intermediate states. We recall that for $4 I>k$, the subspace of 4 -point blocks with $\lambda>m_{k I}$ corresponds to 'unphysical' correlations which violate positivity. Only the 'physical' blocks contribute to $M$ and $\widetilde{M}$.

The form $\widetilde{M}$ corresponds to the diagonal theory over the chiral extension $\mathcal{B}$. Since the local fields of the latter are in general non-diagonal with respect to $\mathcal{A}$, the form $\widetilde{M}$ is a non-diagonal matrix in the $s$-channel basis of conformal block functions which diagonalizes $M$. The ratios of the diagonal elements of the form $\widetilde{M}$ to the corresponding eigenvalues (1.1) of $M$ are invariant under rescaling of the 4-point blocks and thus provide a basisindependent characteristics of the non-diagonal theory associated with the form $\widetilde{M}$. Such ratios were already considered in the above-mentioned pioneer work by Pasquier [2], and have later been computed for specific conformal embeddings [12]. We shall provide in Sect. 3 below an independent computation using previous work on monodromy representations of the braid group $[13,14]$.

Let us turn to the subfactor point of view. As we shall see, one can characterize a local field extension $\mathcal{B}$ of a given theory $\mathcal{A}$ in terms of a triple $(\varrho, W, X)$. Here $\varrho$ is a localized endomorphism of $\mathcal{A}$ equivalent to a reducible representation $\pi$ of $\mathcal{A}$ (the restriction of the vacuum representation of $\mathcal{B}), W$ is an isometric observable (i.e., $W^{*} W=1 l$ ) such that $E=W W^{*}$ projects onto the vacuum representation $\pi_{0}$ of $\mathcal{A}$ contained in $\pi$, and $X$ is a second isometry satisfying a system of identities with $W$, involving $\varrho$, which guarantees the possibility to recover the local extension from these data. The states in the non-trivial subsectors of $\pi$ are created from the vacuum by the extending fields. The operator $X$ may be considered as a generating functional for all the relevant 'generalized Clebsch-Gordan 
coefficients' associated with the inclusion. The mathematical concept behind this notion is a 'harmonic analysis' for subfactors, which generalizes the ordinary harmonic analysis in the case of a compact gauge symmetry. The coefficients determine both the truncated operator product expansions and the amplitudes of 'partial waves' in correlation functions of local charged fields. These partial waves of the subfactor harmonic analysis will be identified with the conformal blocks in chiral current algebra models, and the ClebschGordan coefficients coincide with the structure constants entering the quadratic forms as discussed before.

It is important to note that also in this general context, there is always a 'standard' extension (corresponding to the generic braid-invariant quadratic form $M$ in the case of chiral current algebras) which can be used to fix the normalizations, i.e., to absorb the uncontrolled kinematical model characteristics, by computing invariant double ratios of amplitudes.

Our article is organized as follows. We review in Sect. 2 the monodromy representation of the mapping class group $\mathfrak{B}_{4}$ of the 2 -sphere with 4 punctures in the space of solutions of the KZ equation, and write down the generic braid invariant form corresponding to the $A$ series in the $A D E$ classification. In Sect. 3, the explicit computations are done for two models of special interest, the $E_{\text {even }}$ series conformal embeddings labelled $E_{6}$ and $E_{8}$.

In Sect. 4, we turn to the theory of subfactors (of finite index) and introduce some of the basic concepts which are of particular relevance for the application to (local) field extensions. In Sect. 5, the connection with chiral vertex operators is established, and the general method to compute relative structure constants in terms of subfactors is presented. The method is then applied to the $E_{6}$ inclusion and reproduces the results obtained in Sect. 3 .

The two parts consisting of Sects. 2,3 and Sects. 4,5, respectively, are to a large extent independent of each other. The reader may start with either part according to personal preference. Our point is the comparison of the conceptually different guises under which the same quantities arise in the two approaches.

\section{Braid invariant positive forms in the space of 4-point blocks}

We start with the algebra of observables $\mathcal{A}_{k}=\mathcal{A}_{k}\left(A_{1}\right)$ generated by the level $k$ chiral $s u(2)$ currents. It includes the chiral Sugawara stress-energy tensor. The primary chiral vertex operators $V_{I}[15]$ which intertwine the vacuum sector with the superselection sector of charge $I$ ( $=$ positive energy representation of $\mathcal{A}_{k}$ with lowest energy eigenstates of isospin $I$ ) are assumed to have homogeneous local commutation relations with the currents ('local gauge covariance') and with the stress-energy tensor tensor ('reparametrization covariance'). These assumptions imply the KZ equation [16] as well as the relation between isospin and conformal (scaling) dimension $\Delta_{I}$

$$
(k+2) \Delta_{I}=I(I+1) \quad(2 I=0,1, \ldots k) .
$$

\section{A. The mapping class group and its monodromy representations}

We consider 4-point functions for four primary fields of isospin $I$. We first construct

the $2 I+1$ dimensional representation of the mapping class group $\mathfrak{B}_{4}$ of the 2 -sphere with 4 punctures acting in the $(2 I+1)$-dimensional space of all 4 -point solutions of the 
corresponding $\mathrm{KZ}$ equation, into which the level $k$ enters only via the complex phase

$$
q=\exp \left(\frac{i \pi}{k+2}\right)
$$

Unless $k$ is a positive integer, this space of solutions violates the positivity of correlation functions, and the representation of $\mathfrak{B}_{4}$ is not unitarizable. Yet, it is computationally advantageous to deal with generic $q$ in a first step. At a given level $k \in \mathbb{N}$, positivity is still violated for $4 I>k$, and one has therefore, in a second step, to restrict to the $\left(m_{k I}+1\right)$-dimensional invariant 'physical' subspace spanned by the $s$-channel blocks $s_{\lambda}^{(I)}$ with $\lambda$ in the range of (1.1).

The (projectively represented) mapping class group $\mathfrak{B}_{4}$ can be identified as the braid group of 4 strands on the sphere with generators $B_{i}, i=1,2,3$, such that

$$
\begin{gathered}
B_{1} B_{3}=B_{3} B_{1}, \quad B_{i} B_{i+1} B_{i}=B_{i+1} B_{i} B_{i+1} \quad(i=1,2) \\
B_{1} B_{2} B_{3}^{2} B_{2} B_{1}=B_{3} B_{2} B_{1}^{2} B_{2} B_{3}=q^{-4 I(I+1)}
\end{gathered}
$$

satisfying the additional relation

$$
\left(B_{1} B_{2} B_{3}\right)^{4}=q^{-8 I(I+1)} .
$$

(In the standard definition of $\mathfrak{B}_{4}$, the relations (2.4) and (2.5) are assumed to hold with $q=1$; here we are dealing with a projective representation, or equivalently, with a central extension of the mapping class group.) It can be proven, using only the above relations, that the monodromy operators $B_{1}^{2}$ and $B_{3}^{2}$ are equal. It then follows from (2.4) that the 'fusion' matrix $F$ has square 1:

$$
B_{1} B_{2} B_{1} \equiv B_{2} B_{1} B_{2}=:(-1)^{2 I} q^{-2 I(I+1)} F, \quad F^{2}=1
$$

$F$ plays the role of a $6 j$ symbol (in general, for 4-point blocks of different isospins $I_{i}$, its matrix elements require 6 labels $\left.F_{\lambda \mu}=F_{\lambda \mu}^{I_{1} I_{2} I_{3} I_{4}}\right)$.

An analysis of the solutions of the KZ equation shows that (in the case at hand with four equal isospins $I$ ), actually the generators $B_{1}$ and $B_{3}$ coincide:

$$
B_{1}=B_{3}
$$

Moreover, there exists a basis of solutions [13] for which the fusion matrix has only non-zero elements on the second diagonal,

$$
F_{\lambda \mu}=\delta_{\lambda+\mu, 2 I} \quad(\lambda, \mu=0,1, \ldots 2 I),
$$

while $B_{1}$ is upper triangular:

$$
\left(B_{1}\right)_{\lambda \mu}=(-1)^{2 I-\mu} q^{\mu(\lambda+1)-2 I(I+1)}\left[\begin{array}{c}
2 I-\lambda \\
\mu-\lambda
\end{array}\right] .
$$

Here, $\left[\begin{array}{c}n \\ m\end{array}\right]$ are the (real) $q$-binomial coefficients vanishing for $n<m$ and otherwise given by

$$
\begin{gathered}
{\left[\begin{array}{c}
n \\
m
\end{array}\right]=\frac{[n] !}{[m] ![n-m] !}, \quad[n] !=[n][n-1] !, \quad[0] !=1} \\
{[n]=\frac{q^{n}-q^{-n}}{q-q^{-1}}=\frac{\sin \frac{n \pi}{k+2}}{\sin \frac{\pi}{k+2}}}
\end{gathered}
$$


We are using a non-unitary basis (even for $4 I \leq k$ when $B_{1}$ is unitarizable) which has the following advantages:

(i) it exhibits no singularities for $4 I \geq k+2(2 I \leq k, q$ given by $(2.2))$;

(ii) the entries of the braid matrices and of the invariant forms are elements of the cyclotomic field $\mathbb{Q}\left(q^{1 / 2}\right)$ (or $\mathbb{Q}(q)$ for integer $I ; q^{k+2}=-1$ ).

We anticipate here, that the ratios of structure constants we are finally interested in (eqs. (3.8), (3.9), and (3.15) below) turn out rational and are therefore invariant under Galois automorphisms $q \mapsto q^{n}$ ( $n$ and $2 k+4$ coprime) of this field.

The second generator, $B_{2}$, of $\mathfrak{B}_{4}$ is a conjugate to $B_{1}$ by $F$ :

$$
B_{2}=F B_{1} F \quad\left(B_{1}=F B_{2} F\right)
$$

and appears as a lower triangular matrix.

It is noteworthy that this monodromy representation of $\mathfrak{B}_{4}$ can in fact be derived without a detailed study of the solutions of the $\mathrm{KZ}$ equations. Indeed, the eigenvalues of $B_{1}$ are already read off the 3-point block functions, which are just powers of the coordinate differences. In a basis in which the fusion matrix $F$ has the form (2.8) and $B_{1}$ is upper triangular, the non-diagonal entries of $B_{1}$ and the matrix $B_{2}$ are determined by (2.6) up to a rescaling of the basis. As it was already noted, the ratios of interest will turn out to be invariant under such a rescaling, too.

\section{B. The generic $\mathfrak{B}_{4}$ invariant symmetric form}

The local 4-point function of the two-dimensional theory is defined by a hermitian braid invariant form $M$ in the space of 4-point blocks:

$$
\left\langle\Phi_{I} \Phi_{I} \Phi_{I} \Phi_{I}\right\rangle \propto G_{4}=\sum_{\lambda \mu} \bar{f}_{\lambda} M_{\lambda \mu} f_{\mu} \quad \text { with } \quad M^{+}=M=B^{+} M B \quad\left(B \in \mathfrak{B}_{4}\right)
$$

where an appropriate power of the coordinate differences has been split off as usual, and

$f$ resp. $\bar{f}$ depend only on the conformally invariant cross ratios of coordinate differences on the left- resp. right-moving light-cone. (For further details on the choice of basis $f_{\lambda}$ see [13].)

The above non-unitary realization of $B_{i}$ has the advantage that the inverse generators are just given by the complex conjugate matrices

$$
B_{i}^{-1}=\bar{B}_{i} \quad \text { since } \quad \bar{q}=q^{-1} .
$$

The same is trivially true for $F$.

We are thus looking for a real symmetric form $M=\left(M_{\lambda \mu}\right)={ }^{t} M$ satisfying the braid invariance condition

$$
{ }^{t} B_{i} M=M B_{i} \quad(i=1,2) .
$$

Proposition 2.1: $[14]$ For every $q \neq 0$ there exists a diagonalizable $\mathfrak{B}_{4}$ invariant symmetric form in the space of 4-point solutions of the KZ equation with four isospins $I$

$$
M={ }^{t} S D S \quad \text { where } \quad D_{\lambda \mu}=D_{\lambda} \delta_{\lambda \mu} .
$$

At the values $q=e^{\frac{i \pi}{k+2}}(k \in \mathbb{N})$, the diagonal matrix $D$ has $m_{k I}+1$ non-zero elements (with $m_{k I}$ given by (1.1)):

$$
D_{\lambda} \equiv D_{\lambda}^{(k, I)}=\left\{\frac{[\lambda] ![2 I+1+\lambda] !}{[2 I+1] ![2 \lambda] !}\right\}^{2} \frac{1}{[2 \lambda+1]}, \quad\left(\lambda=0, \ldots m_{k I}\right)
$$


If $4 I>k$, then $D_{\lambda}$ vanish for $m_{k I}<\lambda \leq 2 I$. The transformation matrix $S$ is a real upper triangular matrix with elements

$$
S_{\lambda \mu}=(-1)^{\mu-\lambda}\left[\begin{array}{l}
\mu \\
\lambda
\end{array}\right] \frac{[2 I-\lambda] ![2 \lambda+1] !}{[2 I-\mu] ![\lambda+\mu+1] !} \quad \text { for } \quad 0 \leq \lambda \leq \mu \leq m_{k I}
$$

and $S_{\lambda \mu}=\delta_{\lambda \mu}$ for $\lambda>m_{k I}$.

Sketch of a proof: We consider the similarity transformation

$$
B \mapsto B^{(s)}:=S B S^{-1} .
$$

The specific block form $S=\left(\begin{array}{cc}\Sigma & \Sigma^{\prime} \\ 0 & 1\end{array}\right)$ - where $\Sigma$ is given by (2.18) and the rectangular block $\Sigma^{\prime}$ is only present when $4 I>k$ - implies the block form of the inverse matrix $S^{-1}=\left(\begin{array}{cc}\Sigma^{-1} & -\Sigma^{-1} \Sigma^{\prime} \\ 0 & 1\end{array}\right)$ with

$$
S_{\lambda \mu}^{-1}=\Sigma_{\lambda \mu}^{-1}=\left[\begin{array}{c}
\mu \\
\lambda
\end{array}\right] \frac{[2 I-\lambda] ![\lambda+\mu] !}{[2 I-\mu] ![2 \mu] !} \quad \text { for } \quad 0 \leq \lambda \leq \mu \leq m_{k I}
$$

The transformation (2.19) brings $B_{1}$ in a reduced form for $4 I>k$ and diagonalizes it for $4 I \leq k ;$ in both cases

$$
\left(B_{1}^{(s)}\right)_{\lambda \mu}=\delta_{\lambda \mu}(-1)^{2 I-\lambda} q^{\lambda(\lambda+1)-2 I(I+1)} \quad \text { for } \quad \lambda, \mu \leq m_{k I} .
$$

In particular, the basis $s_{\lambda}=S_{\lambda \mu} f_{\mu}$ of conformal blocks has definite $B_{1}$ monodromy on the physical subspace $0 \leq \lambda \leq m_{k I}$. (For this reason we call $s_{\lambda}$ the $s$-channel basis.)

It follows that $B_{1}^{(s)}$ commutes with $D$ and hence $(2.15)$ holds for $i=1$. Verification of invariance of $M$ with respect to $B_{2}$ or $F$ requires more work. One should either use the explicit form of $M$ :

$$
M_{\lambda \mu}=\frac{(-1)^{\lambda+\mu}[\lambda] ![\mu] !}{[2 I-\lambda] ![2 I-\mu] ![2 I+1] !^{2}} \sum_{\nu=0}^{m_{k I}} \frac{[2 I+\nu+1] !^{2}[2 I-\nu] !^{2}[2 \nu+1]}{[\lambda+\nu+1] ![\mu+\nu+1] ![\lambda-\nu] ![\mu-\nu] !}
$$

or transform $F$ to the $s$-channel basis $\left(F \mapsto F^{(s)}=S F S^{-1}\right)$ - see below.

Remarks: $\triangleright$ An expression of the type (2.16), (2.22) for the invariant form was first derived in $[14$, Sect. 6] using quantum group techniques. The present formulae differ slightly because of a different normalization of the basis. They are related by $[2 I+1]^{2} M_{\lambda \mu}=$ $\left[\begin{array}{c}2 I \\ \lambda\end{array}\right]\left[\begin{array}{c}2 I \\ \mu\end{array}\right] Z_{\lambda \mu}$. Such a change of basis does not affect the ratios of structure constants to be computed below.

$\triangleright$ The Proposition explicitly provides the transition matrix to the $s$-channel basis, from which, together with the spectrum (2.21) of the braid matrix, all the basis-independent quantities of interest in the sequel will be obtained by direct computations.

The braid invariant 2D 4-point function now assumes a diagonal form in the physical $s$-channel basis $s_{\lambda}$ with $\lambda \leq m_{k I}$

$$
G_{4}=\sum_{\lambda=0}^{m_{k I}} D_{\lambda}^{(k, I)} \bar{s}_{\lambda} s_{\lambda}
$$

Summing up we see that, at the quantized values (2.2) of $q$, and more generally for any $q$ such that $q^{k+2}=-1$, the $(2 I+1)$-dimensional representation $\mathfrak{B}_{4}$ of the mapping class 
group is reducible when $4 I>k$. It is also non-unitarizable, the generators $B_{i}$ being not diagonalizable (for $4 I \geq k+2$ ). It is the kernel of the form $M$ that carries a non-unitary factor representation. The $\left(m_{k I}+1\right)$-dimensional subrepresentation $\mathfrak{B}_{4}^{(k, I)}$ preserves a non-degenerate positive form $(2.23)$ and is hence unitarizable. The resulting $\left(m_{k I}+1\right)$ dimensional representation may, in general, still be reducible. As we shall see in Sect. 3A., this fact is responsible for the possible existence of non-diagonal local extensions.

The $s$-channel reflection matrix $F^{(s)}$ (which is related to the exchange of the factors 1 and 3 in (2.13) and which, for four generic isospins, encodes the entire fusion information of the model) is, not surprisingly, considerably more complicated than the original expression (2.8). We have computed it from

$$
F^{(s)}=S F S^{-1}=S U^{-1}=U S^{-1}
$$

in terms of the above $s$-channel transition matrix $S$ which diagonalizes $B_{1}$, and the $u$ channel transition matrix $U=S F$ which diagonalizes $B_{2}$ :

$$
U_{\lambda \mu}=S_{\lambda, 2 I-\mu}=(-1)^{2 I-\lambda-\mu}\left[\begin{array}{c}
2 I-\mu \\
\lambda
\end{array}\right] \frac{[2 \lambda+1] ![2 I-\lambda] !}{[\mu] ![2 I+\lambda-\mu+1] !}
$$

giving

$$
F_{\lambda \mu}^{(s)}=\frac{[\mu] ![2 \lambda+1] ![2 I-\lambda] !}{[\lambda] ![2 \mu] ![2 I-\mu] !} \sum_{\nu=0}^{\mu} \frac{(-1)^{2 I-\lambda+\nu}[\mu+\nu] ![2 I-\nu] !^{2}}{[\nu] !^{2}[\mu-\nu] ![2 I-\lambda-\nu] ![2 I+\lambda-\nu+1] !} .
$$

We note that, even if we use expressions (2.18) and (2.20) beyond the range of their validity (i.e., for $\mu>m_{k I}$ when $4 I>k$ ) where some of the entries of the transition matrix $S$ and $S^{-1}$ are ill defined at the value (2.2) of $q$, the $F$ matrix (2.24) is finite in the physical range $0 \leq \lambda, \mu \leq m_{k I}$. Moreover, the restricted $\left(m_{k I}+1\right) \times\left(m_{k I}+1\right)$ matrices $B_{1}^{(s)}, F^{(s)}$, and

$$
B_{2}^{(s)}=F^{(s)} B_{1}^{(s)} F^{(s)} \quad\left(F^{(s) 2}=1\right)
$$

still satisfy the relations $(2.3)-(2.7)$. This is a non-trivial statement for $4 I>k$.

The braid invariance of the two-dimensional Green's function (2.23) implies the relation

$$
F_{\lambda \mu}^{(s)} D_{\lambda}^{(k, I)}=D_{\mu}^{(k, I)} F_{\mu \lambda}^{(s)}
$$

with the positive eigenvalues $D$ of the form $M$ given by (2.17). Hence on the one hand, the $s$-channel $F$ matrix is symmetrizable, and on the other hand, the ratios of amplitudes for the diagonal extension are given by

$$
\frac{N_{\lambda}^{2}}{N_{\mu}^{2}} \equiv \frac{D_{\lambda}}{D_{\mu}}=\frac{F_{\mu \lambda}^{(s)}}{F_{\lambda \mu}^{(s)}}
$$

\section{Ratios of structure constants for the $E_{6}$ and the $E_{8}$ models}

The braid-invariant 4-point functions (2.13), (2.23) give the monodromy free Green's functions for the 2D local extensions of the chiral su(2) current algebras $\mathcal{A}_{k}$ corresponding to the $A_{k+1}$ series in the $A D E$ classification.

There exists an infinite set of extensions of the $s u(2)$ current algebras for level $k$ a multiple of 4 , corresponding to the $D_{2 n}$ series $(2 n=k / 2+2)$. In these models, the chiral algebras 
are extended by an $\mathcal{A}_{k}$-primary simple current: a Bose field of isospin and conformal dimension

$$
I=\frac{k}{2} \quad \text { and } \quad \Delta_{I}=\frac{I(I+1)}{k+2}=\frac{k}{4} \in \mathbb{N} .
$$

The inclusion of the (nets of) algebras $\mathcal{A}_{k}$ in the resulting field algebras are well understood: it is of the DHR type $[5,17]$ with a global $\mathbb{Z}_{2}$ gauge group which singles out the 'observables' $\mathcal{A}_{k}$ as the gauge invariant elements [18] (for a recent review and further references see [19]).

Here we shall deal with the more interesting exceptional extensions corresponding to conformal embeddings [10]. These are not of the DHR type, i.e., the $\mathcal{A}_{k}$ subalgebras are not the gauge invariants with respect to some global gauge group.

\section{A. Pairs of braid invariant quadratic forms for exceptional embeddings}

There are just two non-trivial chiral extensions of $\mathcal{A}_{k}\left(A_{1}\right)$ corresponding to the conformal embeddings

$$
\begin{aligned}
& \mathcal{A}_{10}=\mathcal{A}_{10}\left(A_{1}\right) \subset \mathcal{A}_{1}\left(B_{2}\right)=\mathcal{B}_{10} \\
& \left.\mathcal{A}_{28}=\mathcal{A}_{28}\left(A_{1}\right) \subset \mathcal{A}_{1}\left(G_{2}\right)=\mathcal{B}_{28}\right)
\end{aligned}
$$

where the labels $E_{6}$ and $E_{8}$ refer to the $E$ series of the $A D E$ classification [3]. The superselection structure of the observables in the 'diagonal' representation space of the respective field extensions is encoded in the exceptional partition functions

$$
\begin{gathered}
Z\left(E_{6}\right)=\left|\chi_{1}+\chi_{7}\right|^{2}+\left|\chi_{4}+\chi_{8}\right|^{2}+\left|\chi_{5}+\chi_{11}\right|^{2} \\
Z\left(E_{8}\right)=\left|\chi_{1}+\chi_{11}+\chi_{19}+\chi_{29}\right|^{2}+\left|\chi_{7}+\chi_{13}+\chi_{17}+\chi_{23}\right|^{2}
\end{gathered}
$$

where the subscripts on the modular characters $\chi$ stand for the dimensions, $2 I+1$, of the $S U(2)$ representations labelling the superselection sectors of $\mathcal{A}_{k}$. Every term in these sums corresponds to a superselection sector of the extended chiral current algebra $\mathcal{B}$, and every sum of modular characters appearing in each term determines the branching of the corresponding sector upon restriction to $\mathcal{A}_{k}$. In particular, the first term added to the vacuum character $\chi_{1}$ in (3.2) corresponds to the $\Delta_{I}=1$ sector of $\mathcal{A}_{k}$ generated by the $\mathcal{B}_{k}$ currents orthogonal to the $\mathcal{A}_{k}$ currents. These are the ( 7 component) $I=3$ primary fields for the $\mathcal{A}_{10}$ theory in the $E_{6}$ case, and the (11 component) $I=5$ primary fields for the $\mathcal{A}_{28}$ theory in the $E_{8}$ case.

The fact that an $\mathcal{A}_{k}$-primary field $\phi_{I}$ (with integer dimension $\Delta_{I}$ ) is a local Bose field in the extended $\mathcal{B}_{k}$ theory means that, in particular, there exists a braid invariant linear combination of 4-point blocks of the associated chiral vertex operators. Namely, the commutation of two fields $\phi_{I}$ corresponds to a monodromy operation on the conformal block functions. In other words, the representation $\mathfrak{B}_{4}^{(k, I)}$ must be reducible and have an invariant subspace of joint eigenvectors of $B_{i}$ with eigenvalue 1 .

In the $s$-channel basis of eq. (2.23), these are combinations of the form

$$
\begin{array}{ccc}
E_{6} & (k=10): & s_{0}^{(3)}+\widetilde{D}_{03} s_{3}^{(3)} \\
E_{8} & (k=28): & s_{0}^{(5)}+\widetilde{D}_{05} s_{5}^{(5)}+\widetilde{D}_{09} s_{9}^{(5)}
\end{array}
$$

where $\widetilde{D}_{\lambda \mu} \equiv \widetilde{D}_{\lambda \mu}^{(k, I)}$ depend on the model, and $\widetilde{D}_{00}=1$ is chosen as a normalization. Twodimensional correlation functions then result as products of two chiral functions (3.3), one for either chiral light-cone. They are thus bilinear in $\left(\bar{s}_{\lambda}, s_{\mu}\right)$ corresponding to a non-diagonal version of $(2.23)$ with $D$ replaced by $\widetilde{D}$ where

$$
\widetilde{D}_{\lambda \mu}=\widetilde{D}_{0 \lambda} \widetilde{D}_{0 \mu}=\widetilde{D}_{\mu \lambda}
$$


The expressions $(3.3)$ are $\mathfrak{B}_{4}^{(k, I)}$-invariant. $B_{1}$-invariance is automatic since all $s$-channel functions $s_{\lambda}^{(I)}$ contributing to (3.3) correspond to the same $B_{1}$ eigenvalue $1\left(=-q^{k+2}\right)$, and it excludes by the same argument all other $s$-channel contributions with $\lambda$ different from 0 or $3\left(E_{6}\right)$ resp. $0,5,9$, or $14\left(E_{8}\right)$. The non-zero elements of $\widetilde{D}$ are determined from $F^{(s)}$ invariance: ${ }^{t} F^{(s)} \widetilde{D}=\widetilde{D} F^{(s)}$. It is sufficient to use the equation

$$
\left({ }^{t} F \widetilde{D}\right)_{0 \mu}=(\widetilde{D F})_{0 \mu}=0 \quad \text { for } \quad \mu=1,2 .
$$

This gives for the isospin $I=3$ current in the $k=10$ model:

$$
\widetilde{D}_{03}=-\frac{F_{01}^{(s)}}{F_{31}^{(s)}}=-\frac{1}{[5]}=-\frac{1}{2+\sqrt{3}} \quad(k=10, I=3)
$$

and for the isospin $I=5$ current in the $k=28$ model:

$$
\widetilde{D}_{05}=\frac{F_{02}^{(s)} F_{91}^{(s)}-F_{01}^{(s)} F_{92}^{(s)}}{F_{51}^{(s)} F_{92}^{(s)}-F_{52}^{(s)} F_{91}^{(s)}}, \quad \widetilde{D}_{09}=\frac{F_{01}^{(s)} F_{52}^{(s)}-F_{02}^{(s)} F_{51}^{(s)}}{F_{51}^{(s)} F_{92}^{(s)}-F_{52}^{(s)} F_{91}^{(s)}} \quad(k=28, I=5)
$$

which can be computed from (2.24).

We note that by a change of scale for the $s$-channel basis functions, $D_{\lambda \mu}$ and $\widetilde{D}_{\lambda \mu}$ change by the same factor, hence their ratios are invariant under rescaling. It is remarkable that these invariant ratios are found to be rational numbers:

$$
\begin{gathered}
\frac{\widetilde{D}_{33}}{D_{33}}=2 \quad(k=10, I=3), \\
\frac{\widetilde{D}_{55}}{D_{55}}=\frac{9}{4}, \quad \frac{\widetilde{D}_{99}}{D_{99}}=\frac{5}{4} \quad(k=28, I=5) .
\end{gathered}
$$

Remark: In a unitary basis in which $D_{\lambda \mu}=\delta_{\lambda \mu}$, the matrix $F^{(s)}$ will become symmetric (and unitary) due to (2.26). This unitarized $\widehat{F}$ can be obtained from our $F$ setting

$$
\widehat{F}_{\lambda \mu}=\left(\operatorname{sign} F_{\lambda \mu}\right) \sqrt{F_{\lambda \mu} F_{\mu \lambda}}
$$

In such a unitary basis, the above ratios will coincide with $\widetilde{D}_{\lambda \lambda}$.

\section{B. The braid group representation in the Ramond sector}

The extended model $\mathcal{B}_{10}=\mathcal{A}_{1}\left(B_{2}\right)$ (see Sect. 3A.) is parallel in many respects to the Ising model and the $s u(2)$ level 2 current algebra theory. All three models have three superselection sectors with identical fusion rules, and involve a simple current of dimension $\Delta=\frac{1}{2}$. For $\mathcal{B}_{10}$, this field is the $S O(5)$ vector field $\psi$ which is also an irreducible $\mathcal{A}_{10}$ primary field of isospin 2 .

The state space of the fermionic field $\psi$ splits into two irreducible representations with respect to the extended 'super current algebra' generated by $\psi(z)$ : the Neveu-Schwarz sector $\mathcal{H}_{1} \oplus \mathcal{H}_{5}$, and the Ramond sector $\mathcal{H}_{2}$, where $\mathcal{H}_{d}$ denote the level $1 \operatorname{spin}(5)$ current algebra representations labelled by the dimension $d$ of their lowest energy subspace. The correlation functions of $\psi$ are single-valued in the Neveu-Schwarz sector, and double valued in the Ramond sector.

Furthermore, in all three models, the primary dimension in the Ramond sector is related to the Virasoro central charge

$$
\Delta=\frac{1}{8} c
$$


$c$ being given as $\frac{1}{2}$ times the number of components of $\psi\left(c=\frac{5}{2}\right.$ for $\left.\mathcal{B}_{10}\right)$.

We proceed to compute the $4 \times 4$ braid matrices in the $s$-channel basis of all $\mathcal{A}_{10}$ conformal blocks of four fields of isospin $I=\frac{3}{2}$ and dimension $\Delta=\frac{5}{16}$ which belong to the Ramond sector of $\mathcal{B}_{10}$ (see eq. $(3.2 a)$ ). Then we determine the subrepresentation acting in the subspace of conformal blocks of the extended theory $\mathcal{B}_{10}$ which constitute the $2 \mathrm{D}$ local Ramond 4-point functions.

Applying (2.21) and (2.24) for $I=\frac{3}{2}$, we obtain

$$
B_{1}^{(s)}=q^{\frac{9}{2}}\left(\begin{array}{cccc}
1 & 0 & 0 & 0 \\
0 & -q^{2} & 0 & 0 \\
0 & 0 & q^{6} & 0 \\
0 & 0 & 0 & 1
\end{array}\right) \quad\left(k=10, q=e^{\frac{i \pi}{12}}, I=\frac{3}{2}\right)
$$

and

$$
F^{(s)}=\left(\begin{array}{cccc}
\frac{1-[3]}{3[2]} & \frac{[3]-1}{3} & -\frac{[3]}{3[2]} & \frac{1}{3} \\
\frac{4-[3]}{3} & -\frac{1}{[2]} & 0 & \frac{2}{3[2]} \\
-\frac{[3]}{2[2]} & 0 & \frac{[3]}{2[2]} & \frac{[3]-1}{6} \\
1 & \frac{[3]}{[2]} & \frac{[3]-1}{3} & \frac{4-[3]}{6[2]}
\end{array}\right)=\left(\begin{array}{cccc}
\frac{1-\sqrt{3}}{\sqrt{6}} & \frac{1}{\sqrt{3}} & -\frac{\sqrt{2}}{3} & \frac{1}{3} \\
\frac{\sqrt{3}-1}{\sqrt{3}} & \frac{1-\sqrt{3}}{\sqrt{2}} & 0 & \sqrt{2} \frac{\sqrt{3}-1}{3} \\
-\frac{1}{\sqrt{2}} & 0 & \frac{1}{\sqrt{2}} & \frac{1}{2 \sqrt{3}} \\
1 & \sqrt{2} & \frac{1}{\sqrt{3}} & \frac{2-\sqrt{3}}{\sqrt{6}}
\end{array}\right) .
$$

The first matrix displayed here was computed with $q$-number identities valid for every Galois transform of $q$. Evaluating $[3]=\sqrt{2}[2]=1+\sqrt{3}$ at $q=e^{\frac{i \pi}{12}}$, one obtains the second matrix (3.13).

We are now looking for an $E_{6}$-type braid invariant $s$-channel quadratic form $\widetilde{D} \equiv \widetilde{D}^{(10,2)}$

$$
\widetilde{D}=\left(\begin{array}{cccc}
1 & 0 & 0 & \widetilde{N}_{3} \\
0 & 0 & 0 & 0 \\
0 & 0 & \widetilde{N}_{2}^{2} & 0 \\
\widetilde{N}_{3} & 0 & 0 & \widetilde{N}_{3}^{2}
\end{array}\right) \quad \text { where } \quad \widetilde{N}_{\lambda}=\frac{\widetilde{N}_{\frac{3}{2}} \frac{3}{2} \lambda}{\widetilde{N}_{\frac{3}{2}} \frac{3}{2} 0}
$$

The equality of the first and the last eigenvalue of $B_{1}^{(s)}$ (eq. (3.12)) ensures $B_{1}$-invariance of $\widetilde{D}$. The real parameters $\widetilde{N}_{\lambda}$ can be determined from $F$-invariance ${ }^{t} F \widetilde{M}=\widetilde{M} F$ of the quadratic form $\widetilde{M}={ }^{t} S \widetilde{D} S$, which implies

$$
F_{01}^{(s)}+F_{31}^{(s)} \widetilde{N}_{3}=0, \quad F_{20}^{(s)} \widetilde{N}_{2}^{2}=F_{02}^{(s)}+\widetilde{N}_{3} F_{32}^{(s)}
$$

This yields $\widetilde{N}_{3}=-1 / \sqrt{6}$ and $\widetilde{N}_{2}^{2}=1$ for $I=\frac{3}{2}$. We obtain the invariant ratios with the structure constants $D_{\lambda} \equiv N_{\lambda}^{2}$ of the diagonal theory given by (2.17) or by (2.27):

$$
\frac{\widetilde{N}_{3}^{2}}{N_{3}^{2}}=\frac{F_{10}^{(s)} F_{01}^{(s)}}{F_{13}^{(s)} F_{31}^{(s)}}=\frac{1}{2}, \quad \frac{\widetilde{N}_{2}^{2}}{N_{2}^{2}}=1-\frac{F_{01}^{(s)} F_{32}^{(s)}}{F_{02}^{(s)} F_{31}^{(s)}}=\frac{3}{2} \quad\left(k=10, I=\frac{3}{2}\right) .
$$

The same result is obtained for the invariant ratio of structure constants for the isospin $I=\frac{7}{2}$ field, as expected since the latter is the 'partner' of the isospin $I=\frac{3}{2}$ field in the partition function $(3.2 a)$, related by the simple current of isospin 5 . Indeed, according to $(2.1)$,

$$
\Delta\left(\frac{7}{2}\right)-\Delta\left(\frac{3}{2}\right)=\frac{21}{16}-\frac{5}{16}=1
$$

and hence the matrices $B_{1}^{(s)}$ (projected into the physical subspace of $s$-channel blocks $s_{\lambda}$, $\left.0 \leq \lambda \leq m_{k I}\right)$ coincide for $I=\frac{3}{2}$ and $\frac{7}{2}$. It is instructive to verify that, although the $s$-channel $F$-matrices do not coincide for $I=\frac{7}{2}$ and $\frac{3}{2}$, the invariant ratios (3.15) are the same. 
In computing $F^{(s)}=U S^{-1}$ for $I=\frac{7}{2}$ in terms of the $s$ - and $u$-channel transition matrices $S$ and $U$ (see Sect. 2), one encounters the problem of the reduction from the 8-dimensional space of KZ solutions to the 4-dimensional physical subspace. It is simplified by the observation that due to the triangular form of $S$ and $U$, the reduced matrix $F^{(s)}$ for $4 I>k$ is obtained by just taking the first $m_{k I}+1=k-2 I+1$ rows and columns of both $U$ and $S^{-1}$. In particular, for $I=\frac{7}{2}$ we observe that the symmetrized (unitary) matrices (3.10) corresponding to $I=\frac{3}{2}$ (eq. (3.13)) and to $I=\frac{7}{2}$ coincide.

The 2-dimensional braid invariant subspace comprising the conformal blocks of local Ramond fields of the $\mathcal{B}_{10}$ model is spanned by the pair of vectors

$$
v_{0}=\left(-\frac{2}{3}, 0,0, \sqrt{\frac{2}{3}}\right), \quad v_{2}=(0,0,1,0)
$$

which are ortho-normalized with respect to the metric (3.14):

$$
{ }^{t} v_{a} \widetilde{D} v_{b}=\delta_{a b} \quad(a, b=0,2) .
$$

In this basis, we have the following reduced form of the $s$-channel generators

$$
\hat{B}_{1}^{(s)}=q^{\frac{3}{2}}\left(\begin{array}{cc}
q^{3} & 0 \\
0 & -q^{-3}
\end{array}\right), \quad \hat{F}^{(s)}=-\frac{[2]}{[3]}\left(\begin{array}{cc}
-1 & 1 \\
1 & 1
\end{array}\right) \quad\left(k=10, I=\frac{3}{2}\right) .
$$

(At $q=e^{\frac{i \pi}{12}}$, one has $[3]=\sqrt{2}[2]$ ). Identical expressions are obtained for the reduced generators acting in the invariant subspace of conformal blocks for $I=\frac{7}{2}$.

The resulting 2-dimensional representation of $\mathfrak{B}_{4}$ is a finite matrix group. It is a central extension of the 24-element 2-fold covering of the tetrahedron group. This is worth noticing, since the appearance of finite matrix groups among the monodromy representations of $\mathfrak{B}_{4}$ is rather exceptional [20].

\section{Subfactors for field extensions}

We turn now to the treatment of the same problem: the determination of relative amplitudes like (3.8), (3.9), in the algebraic (DHR) framework of quantum field theory. A theory $\mathcal{A}$ is described by a local net of von Neumann algebras $\mathcal{A}(\mathcal{O})$ of observables in the space-time region $\mathcal{O}$, which generate the global $C^{*}$ algebra $\mathcal{A}$. These regions may be double cones $(\mathcal{O})$, or intervals $(\mathcal{J})$ on the light-cone in chiral conformal theories.

In the following, we consider a pair of local quantum field theories given by the nets of local von Neumann algebras $\mathcal{A}(\mathcal{O})$ and $\mathcal{B}(\mathcal{O})$ such that

$$
\mathcal{A}(\mathcal{O}) \subset \mathcal{B}(\mathcal{O})
$$

are irreducible inclusions with common unit. Our terminology will be 'observables' for $a \in \mathcal{A}$ and 'charged fields' for $b \in \mathcal{B}$. We have in mind two specific such nets, namely

1. the conformal inclusion $[12,21]$ of the chiral $s u(2)$ current algebra at level 10 into the chiral $s p(4)$ current algebra at level 1, denoted by

$$
\mathcal{A}_{c h}(\mathcal{J}) \subset \mathcal{B}_{c h}(\mathcal{J})
$$

where $\mathcal{J}$ are intervals on the circle (= compactified conformal light-cone), and 
2. the two-dimensional WZNW model [11] of the chiral su(2) currents at level 10 (on both light-cones) contained in the algebra of two-dimensional local fields constructed by diagonal contraction of chiral vertex operators (exchange fields):

$$
\mathcal{A}^{(2)}(\mathcal{O}) \equiv \mathcal{A}_{c h}(\mathcal{J}) \otimes \mathcal{A}_{c h}(\overline{\mathcal{J}}) \subset \mathcal{F}^{(2)}(\mathcal{O})
$$

where a two-dimensional double cone $\mathcal{O}=\mathcal{J} \times \overline{\mathcal{J}}$ is the Cartesian product of two chiral light-cone intervals.

Note that the model (4.3) is the one described by the standard diagonal form $D$ in the previous sections, while the form $\widetilde{D}$ corresponds to a combination of (4.2) and (4.3):

$$
\mathcal{A}^{(2)}(\mathcal{O}) \equiv \mathcal{A}_{c h}(\mathcal{J}) \otimes \mathcal{A}_{c h}(\overline{\mathcal{J}}) \subset \mathcal{B}_{c h}(\mathcal{J}) \otimes \mathcal{B}_{c h}(\overline{\mathcal{J}}) \subset \widetilde{\mathcal{F}}^{(2)}(\mathcal{O}) .
$$

Here, the first inclusion is the tensor product of the chiral extensions (4.2) and the second inclusion is the standard diagonal contraction of chiral vertex operators for $\mathcal{B}_{c h}$. (There will be said more about these 'standard' constructions in Sect. 5; see also [22, 9].)

A subfactor $A \subset B$ is irreducible if the relative commutant is trivial: $A^{\prime} \cap B=\mathbb{C}$. This requirement excludes from our analysis all chiral current subalgebras associated with subgroups, unless the embedding is 'conformal' [10], since the coset stress-energy tensor is contained in the relative commutant. However, including the coset stress-energy tensor into the observables (which then have the structure of a tensor product of two chiral theories), would again yield an irreducible inclusion [27, 9].

We have to recall some subfactor theory. First, we note that we are dealing with type $I I I_{1}$ subfactors, since under very general conditions, the local von Neumann algebras in quantum field theory are hyperfinite type $I I I_{1}$ factors $[23,24]$. Associated with an (irreducible) type $I I I$ subfactor $A \subset B$ is a canonical endomorphism $\gamma \in \operatorname{End}(B)$ such that $\gamma(B) \subset A$ is a dual subfactor $[8,25] . A \subset B$ has finite index if and only if [8] there is a pair of isometries $W \in A$ and $V \in B$ such that the following operator identities hold:

$$
\begin{array}{llc}
\text { (a) } & W a=\varrho(a) W & \left(a \in A, \varrho:=\left.\gamma\right|_{A}\right) \\
\text { (b) } & V b=\gamma(b) V & (b \in B) \\
\text { (c) } & W^{*} V=\lambda^{-1 / 2} l=W^{*} \gamma(V) .
\end{array}
$$

The real number $\lambda$ is called the index of the subfactor $A \subset B$. These relations express the duality between $A \subset B$ and $\gamma(B) \subset A$. They also state that $B$ is the Jones extension [1] of $A$ by its subfactor $\gamma(B)$. The Jones projection is $E=V V^{*}$, satisfying the JonesTemperley-Lieb relation with its dual $F=W W^{*}$ :

$$
E F E=\lambda^{-1} E, \quad F E F=\lambda^{-1} F .
$$

Associated with these data, there is a conditional expectation $\mu: B \rightarrow A$ given by

$$
\mu(b)=W^{*} \gamma(b) W \quad(b \in B),
$$

and conversely the canonical endomorphism can be expressed in the form

$$
\gamma(b)=\lambda \cdot \mu\left(V b V^{*}\right) \quad(b \in B) .
$$

$\mu$ is a positive and $A$-linear map which generalizes the Haar average over a compact group acting on $B$ with fixpoints $A$. It satisfies the Pimsner-Popa bound

$$
\mu(b) \geq \lambda^{-1} \cdot b \quad(b \in B, b \geq 0)
$$


as an operator estimate for every positive operator $b \in B$. This lower bound for conditional expectations was first introduced in [26] to define the index. It is optimal since it is saturated by

$$
\mu\left(V V^{*}\right)=\lambda^{-1} 1 .
$$

We note also that $W=\lambda^{-1 / 2} \cdot \mu(V)$. The physical relevance of these objects will become clear in due context.

The following results on quantum field theoretical nets of subfactors as in eq. (4.1) will be proven (and qualified) elsewhere [9]. Let us just state the essentials. Let the vacuum vector $\Omega$ be cyclic and separating for every local von Neumann algebra $\mathcal{B}(\mathcal{O})$ of the theory $\mathcal{B}$, i.e. $\pi^{0}(\mathcal{B}(\mathcal{O})) \Omega$ are dense subspaces of the vacuum representation space $\mathcal{H}^{0}$. This property holds, by the Reeh-Schlieder Theorem, quite generally for covariant quantum field theories with positive energy. Let also $\mathcal{H}_{0}=\overline{\pi^{0}(\mathcal{A}) \Omega} \subset \mathcal{H}^{0}$ be the vacuum representation space of $\mathcal{A}$ such that $\Omega$ is also cyclic and separating in $\mathcal{H}_{0}$ for every $\mathcal{A}(\mathcal{O})$. Let furthermore the conditional expectation $\mu$ preserve localizations, i.e., map $\mathcal{B}(\mathcal{O})$ onto $\mathcal{A}(\mathcal{O})$. If the local subfactors are irreducible and therefore possess a unique conditional expectation, then $\mu$ must commute with the translations $(=$ the rotations of the circle in the case of a chiral conformal theory). If the vacuum state $\omega=\left\langle\Omega, \pi^{0}(\cdot) \Omega\right\rangle$ on $\mathcal{B}$ is the unique translation invariant state, then it must also be invariant under $\mu$, i.e.,

$$
\omega \circ \mu=\omega \quad \text { on } \mathcal{B} \text {. }
$$

We shall assume the invariance property (4.8) in the sequel. The underlying structure admits the interpretation as a generalized global unbroken gauge symmetry with $\mu$ generalizing the gauge group average [9].

Under these circumstances, the canonical endomorphism $\gamma$ defined above for a fixed local subfactor $\mathcal{A}\left(\mathcal{O}_{0}\right) \subset \mathcal{B}\left(\mathcal{O}_{0}\right)$ extends to an endomorphism of the global $C^{*}$ algebra $\mathcal{B}$, and maps $\mathcal{B}$ into the global $C^{*}$ algebra of observables $\mathcal{A}$. Restricted to the observables, $\left.\gamma\right|_{\mathcal{A}}$ turns out to be a localized endomorphism with localization in $\mathcal{O}_{0}$, denoted by $\varrho$ in the sequel. It therefore describes a (reducible) superselection sector [5] of the theory $\mathcal{A}$. Its physical significance is given by the following

Proposition 4.1: $[27,9]$ Let $\pi_{0}$ denote the vacuum representation of $\mathcal{A}$ on $\mathcal{H}_{0}$, and $\pi^{0}$ the vacuum representation of $\mathcal{B}$ on $\mathcal{H}^{0}$. Then $\pi^{0}$ considered as a reducible representation of the subalgebra $\mathcal{A}$ is unitarily equivalent to the representation $\pi_{0} \circ \varrho$ of $\mathcal{A}$.

In other words: the superselection sector $\varrho$ comprises all the charged sectors of $\mathcal{A}$ which are interpolated from the vacuum by fields in $\mathcal{B}$. If, as endomorphisms, $\varrho \simeq \bigoplus_{s} N_{s} \varrho_{s}$, then as representations,

$$
\left.\pi^{0}\right|_{\mathcal{A}} \simeq \pi_{0} \circ \varrho \simeq \bigoplus_{s} N_{s} \pi_{s}
$$

where $N_{s}$ are finite multiplicities, and $\pi_{s} \equiv \pi_{0} \varrho_{s}$. As is well known, if the observables $\mathcal{A}$ are the gauge invariants under a compact gauge symmetry group of $\mathcal{B}$, then the decomposition (4.9) is given by the representations of the gauge group, with multiplicities $N_{s}$ given by the dimensions of the latter.

Eq. (4.9) allows to compute the index $\lambda$ of the subfactor. It is given by the formula

$$
\lambda=d(\varrho)=\sum_{s} N_{s} d\left(\varrho_{s}\right)
$$


in terms of the statistical dimensions $d\left(\varrho_{s}\right) \equiv d_{s}$ of the superselection sectors [5] contained in $\varrho$. In the gauge group case, $d\left(\varrho_{s}\right)=N_{s}$, and the index equals the order of the group.

In the models (4.2), (4.3), the branching of the vacuum sector of $\mathcal{B}$ is well known, leading to $\varrho \simeq \varrho_{0} \oplus \varrho_{3}$ for the inclusion (4.2) and $\varrho \simeq \bigoplus_{I} \varrho_{I} \otimes \varrho_{I}$ for the inclusion (4.3), where $\varrho_{I}$ are the isopin $I$ sectors of the chiral su(2) current algebra. $\varrho_{0} \equiv i d$ corresponds to the vacuum representation. In the former case, the formula (4.10) yields the index $\lambda=d_{0}+d_{3}=1+\sin \frac{7 \pi}{12} / \sin \frac{\pi}{12}=3+\sqrt{3}$. (For the coincidence of statistical dimensions and 'quantum dimensions' $d\left(\varrho_{I}\right)=[2 I+1]$ for $s u(2)$ current algebras see [27].)

The formulae $(4.4)-(4.7)$ remain valid for $\gamma$ considered as an endomorphism of $\mathcal{B}$ and for $\varrho$ as an endomorphism of $\mathcal{A}$. Note that the isometries $W$ and $V$ are local operators $W \in \mathcal{A}\left(\mathcal{O}_{0}\right)$ and $V \in \mathcal{B}\left(\mathcal{O}_{0}\right)$. We shall refer to the intertwining properties expressed by eqs. $(4.4(a, b))$ by the notation $V: i d \rightarrow \gamma$ and $W: i d \rightarrow \varrho$ in the sequel. The latter implies that $\pi_{0}\left(W W^{*}\right)$ is the projection in the representation space of $\pi_{0} \circ \varrho$ which projects onto the vacuum subrepresentation contained in (4.9).

For every other subsector $\pi_{s}$ contained in (4.9) there are corresponding projections of the form $\pi_{0}\left(W_{s, i} W_{s, i}^{*}\right)$ where $W_{s, i}: \varrho_{s} \rightarrow \varrho$ are orthonormal isometric intertwiners in $\mathcal{A}\left(\mathcal{O}_{0}\right)$; the multiplicity index $i$ runs from 1 to $N_{s}$. For simplicity, we shall in the following consider only multiplicities $N_{s}=1$ (covering abelian gauge groups, as well as our models above). One has the orthogonality relation $W_{s}^{*} W_{t}=\delta_{s t}$ (because otherwise, the intertwiner $W_{s}^{*} W_{t}: \varrho_{t} \rightarrow \varrho_{s}$ would contradict the inequivalence of the representations $\pi_{s}$ and $\pi_{t}$ ), and the completeness relation $\sum_{s} W_{s} W_{s}^{*}=1$. Clearly, $W_{0} \equiv W$.

Putting

$$
\psi_{s}:=W_{s}^{*} V
$$

we obtain charged intertwiners, i.e., elements of $\mathcal{B}$ which satisfy the commutation relations with the observables

$$
\psi_{s} a=\varrho_{s}(a) \psi_{s} \quad(a \in \mathcal{A}) .
$$

This equation means that $\psi_{s} \in \mathcal{B}$ make transitions (in the vacuum representation of $\mathcal{B}$ ) between the vacuum representation of $\mathcal{A}$ and the charged representations $\pi_{s}$.

Conversely,

$$
V=\sum_{s} W_{s} \psi_{s}
$$

and the commutation relation

$$
V a=\varrho(a) V \quad(a \in \mathcal{A})
$$

gives to $V$ the physical interpretation as a 'master field' carrying the reducible charge $\varrho$ from which the charged intertwiners $\psi_{s}$ are projected out by means of $W_{s}$.

A particularly interesting object is the observable operator

$$
X:=\gamma(V) \in \mathcal{A}\left(\mathcal{O}_{0}\right)
$$

From the definitions it is clear that $X$ is an isometric intertwiner $X: \varrho \rightarrow \varrho^{2}$. Indeed, we can compute

$$
X=\gamma(V)=\lambda \mu\left(V V V^{*}\right)=\lambda \sum_{s t u} \mu\left(W_{t} \psi_{t} W_{s} \psi_{s} \psi_{u}^{*} W_{u}^{*}\right)=\lambda \sum_{s t u} W_{t} \varrho_{t}\left(W_{s}\right) \cdot \mu\left(\psi_{t} \psi_{s} \psi_{u}^{*}\right) \cdot W_{u}^{*}
$$

where the expressions $\mu\left(\psi_{t} \psi_{s} \psi_{u}^{*}\right)$ are observable intertwiners $T: \varrho_{u} \rightarrow \varrho_{t} \varrho_{s}$. They are therefore multiples of isometric basis intertwiners $T_{e}$ which project onto the subrepresentations $\pi_{u}$ contained in the DHR composition product $\left.\pi_{t} \times \pi_{s}=\pi_{0} \circ \varrho_{t} \varrho_{s}\right)$ :

$$
\lambda \mu\left(\psi_{t} \psi_{s} \psi_{u}^{*}\right)=\lambda(e) \cdot T_{e}
$$


with coefficients

$$
\lambda(e) \mathbb{l}=\lambda \cdot T_{e}^{*} \mu\left(\psi_{t} \psi_{s} \psi_{u}^{*}\right)
$$

(The multi-index $e$ stands here and in the sequel for the fusion channel $\pi_{u} \prec \pi_{t} \times \pi_{s}$.)

Denoting by $\tilde{T}_{e}=\varrho\left(W_{s}\right) W_{t} \cdot T_{e} \cdot W_{u}^{*}$ the 'lifts' of intertwiners $T_{e}: \varrho_{u} \rightarrow \varrho_{t} \varrho_{s}$ to intertwiners $\tilde{T}_{e}: \varrho \rightarrow \varrho^{2}$, we obtain the expansion

$$
X=\sum_{e} \lambda(e) \tilde{T}_{e}
$$

We note that only channels $e$ contribute to (4.17) for which $\varrho_{s}, \varrho_{t}, \varrho_{u}$ are all subsectors of the canonical endomorphism $\varrho$, in spite of the fact that in general $\varrho_{t} \varrho_{s}$ will also contain subsectors which are not contained in $\varrho$. We shall relate this observation to the 'truncated fusion rules' in the next section.

The importance of the isometry $X$ is due to the following result, while the relevance of its expansion coefficients $\lambda(e)$ will reveal itself in the sequel.

Proposition 4.2: [28] The irreducible subfactor $A \subset B$ is uniquely characterized (up to unitary equivalence) by the triple $(\varrho, W, X)$, where $\varrho \in \operatorname{End}(A)$ and $W: i d \rightarrow \varrho$ and $X: \varrho \rightarrow \varrho^{2}$ are isometric intertwiners in $A$, satisfies the following identities

$$
\begin{array}{lcl}
\text { (i) } & W^{*} X=\lambda^{-1 / 2} l=\varrho\left(W^{*}\right) X \quad \text { with } \quad \lambda=d(\varrho) \\
(\text { ii }) & X X^{*}=\varrho\left(X^{*}\right) X \\
(\text { iii }) & X X=\varrho(X) X .
\end{array}
$$

Clearly, the identities (4.18) follow from (4.4). Conversely, given a triple as in Prop. 4.2, one recovers $B$ as follows. Put $A_{1}:=X^{*} \varrho(A) X$ and $B:=$ the Jones extension of $A$ by $A_{1}$. This extension is of the form $B=A V$ where $V$ is an isometry with $V V^{*}=E$, the Jones projection. Define $\gamma \in \operatorname{End}(B)$ by $\gamma(a V):=\varrho(a) X$. Then $\gamma$, satisfying (4.4), is the canonical endomorphism for $A \subset B$ and $\varrho=\left.\gamma\right|_{A}, A_{1}=\gamma(B)$.

In our present context, $A=\mathcal{A}(\mathcal{O})$ and $B=\mathcal{B}(\mathcal{O})$, the point about this characterization of (4.1) is that it entirely refers to the observables and their superselection sectors. Finding such a triple in a given theory $\mathcal{A}$ amounts to find a field extension $\mathcal{B}$ of the observables of the form (4.1). The problem involves the knowledge of the 'fusion coefficients' of the theory $\mathcal{A}$, i.e., the coefficients of expressions like $\varrho_{v}\left(T_{e}\right)$ (entering $\varrho(X)$ ) in terms of a basis $T_{g} T_{h} T_{f}^{*}$. These are the solutions to the Moore-Seiberg 'pentagon identities' [29] which are intrinsically determined by the DHR theory of superselection sectors [7] (but often tedious to compute).

Let us briefly sketch the 'reverse program' of construction and classification of (local) field extensions of finite index [9].

The main step is to decide which combinations $\varrho \simeq \bigoplus_{s} N_{s} \varrho_{s}$ of the irreducible localized endomorphisms (sectors) of $\mathcal{A}$ are canonical endomorphisms of the local von Neumann algebra $A \equiv \mathcal{A}\left(\mathcal{O}_{0}\right)$ with respect to some subfactor $A_{1} \subset A$. This amounts [28] to verify the existence of a pair of isometric intertwiners $W: i d \rightarrow \varrho$ and $X: \varrho \rightarrow \varrho^{2}$ in $\mathcal{A}\left(\mathcal{O}_{0}\right)$ solving (4.18). If the desired inclusion is required to be irreducible, then $i d \prec \varrho$ with multiplicity $N_{0}=1$, and if the index is finite, then one can prove the bound $N_{s} \leq d_{s}$. Therefore, if $\mathcal{A}$ is a 'rational' theory, i.e., has only finitely many sectors of finite statistics, then the classification problem is a finite problem in the form of a non-linear system for the unknown coefficients $\lambda(e)_{i j}^{k}$ (with multiplicities). 
If we are interested in local field extensions, then we have to require in addition (see below) that the solution $X$ satisfies

$$
\varepsilon_{\varrho} X=X
$$

where $\varepsilon_{\varrho} \in \varrho^{2}(\mathcal{A})^{\prime} \cap \mathcal{A}\left(\mathcal{O}_{0}\right)$ is the statistics operator for the localized endomorphism $\varrho$ [5]. $\varepsilon_{\varrho}=U^{*} \varrho(U)$ can be computed in terms of a charge transporting intertwiner $U: \varrho \rightarrow \varrho$ where $\hat{\varrho}$ is an equivalent endomorphism localized at space-like distance from $\varrho$.

Every solution $(\varrho, W, X)$ to the system (4.18) defines a field net $\mathcal{B}$ extending $\mathcal{A}$ with finite index $\lambda=d(\varrho)$ as follows. If $\varrho$ is localized in $\mathcal{O}_{0}$, one reconstructs $B=\mathcal{B}\left(\mathcal{O}_{0}\right)$ and $\gamma \in \operatorname{End}(B)$ from $A=\mathcal{A}\left(\mathcal{O}_{0}\right)$ as in the remark after Prop. 4.2. Thus $\mathcal{B}\left(\mathcal{O}_{0}\right)=\mathcal{A}\left(\mathcal{O}_{0}\right) V$ for an isometry $V \in \mathcal{B}\left(\mathcal{O}_{0}\right)$ satisfying (4.4). Next, $\mathcal{B}(\mathcal{O}):=\mathcal{A}(\mathcal{O}) U V$ are defined with the help of charge transporters $U \in \mathcal{A}$, i.e., unitary intertwiners $U: \varrho \rightarrow \hat{\varrho}$ where $\hat{\varrho}$ is localized in $\mathcal{O}$. Note that $\mathcal{B}(\mathcal{O})$ thus defined contains the identity operator $1 \propto W^{*} V=\hat{W}^{*} U V$ since $\hat{W}=U W: i d \rightarrow \varrho \hat{~}$ is in $\mathcal{A}(\mathcal{O})$. Consequently, $\mathcal{B}(\mathcal{O})$ contains and extends $\mathcal{A}(\mathcal{O})$. This construction yields a net $\mathcal{B}$ which is relatively local with respect to $\mathcal{A}$, since $\varrho$ is localized; namely if $\mathcal{O}$ is at space-like distance from $\mathcal{O}_{0}$, then $\mathcal{A}\left(\mathcal{O}_{0}\right)$ commutes with $\mathcal{B}(\mathcal{O})$ :

$$
U V \cdot a=U \varrho(a) V=\hat{\varrho}(a) U V=a \cdot U V \quad\left(a \in \mathcal{A}\left(\mathcal{O}_{0}\right)\right) .
$$

The field extension $\mathcal{B}$ turns out to be local if and only if the solution $X$ satisfies also (4.19). Namely, the commutativity of $V \in \mathcal{B}\left(\mathcal{O}_{0}\right)$ with $U V \in \mathcal{B}(\mathcal{O})$ at space-like distance is equivalent to $V V=U^{*} V U V$, and hence to

$$
X V=\gamma(V) V=V V=U^{*} V U V=U^{*} \varrho(U) V V=\varepsilon_{\varrho} \gamma(V) V=\varepsilon_{\varrho} X V .
$$

We observe that the system (4.18) alone will have many solutions, e.g., those of the form $\varrho=\bar{\sigma} \sigma, X=\sigma(\bar{W})$ where $\sigma$ is any irreducible localized endomorphism of the theory $\mathcal{A}$ with finite statisticss, $\bar{W}: i d \rightarrow \sigma \bar{\sigma}$ an isometry. These solutions will, however, not satisfy (4.19) in general, and will therefore not give rise to local field extensions.

Note that, actually, locality of the field net was not required for the general analysis in the first part of this section, as long as it has the Reeh-Schlieder property, and fields commute with observables at space-like distance. However, since it is not clear which physical principles should determine a 'good choice' of a non-local and therefore a priori unobservable field algebra except that it generates the superselection sectors of the observables, we prefer to consider only local field extensions which offer the option to be regarded as observable theories of their own.

If $\mathcal{A}$ are the gauge invariants under a gauge group acting on $\mathcal{B}$, then the system (4.18) has a solution with multiplicities $N_{s}$ given by the dimensions of the representations of the gauge group. The corresponding coefficients $\lambda(e)_{i j}^{k}$ in the expansion (4.17) of $X$ are precisely the Clebsch-Gordan coefficients. Indeed, one may rephrase the content of the Doplicher-Roberts (DR) reconstruction theorem [17] as follows: every system of sectors of the observables which have finite permutation group statistics among each other, closed under composition, reduction, and conjugation, admits a solution to (4.18) with $X$ given by (4.17) in terms of Clebsch-Gordan coefficients of some compact gauge group. The DR solution is distinguished by the validity of (4.19) if there are only bosonic sectors of $\mathcal{A}$, and a graded variant of (4.19) in the presence of fermionic sectors.

We emphasize that, while our general theory above contains the case of a compact gauge symmetry group, the models (4.2), (4.3) we are actually interested in are not given by a gauge symmetry group. The sectors $\pi_{s}$ contained in the restriction $\left.\pi^{0}\right|_{\mathcal{A}}$ are not closed under composition, and their multiplicities differ from their statistical dimensions. Although 
the fields are local, the sectors $\pi_{s}$ have braid group statistics. None of these features could hold with a gauge group.

Displayed in terms of the coefficients $\lambda(e)_{i j}^{k}$, the system (4.18) is converted into a system of identities well-known to hold for Clebsch-Gordan coefficients (with the $6 j$ symbols as fusion coefficients). The absence of a completeness property in (4.18) is related to the truncated fusion rules discussed in the next section.

\section{Truncated fusion rules and partial wave decomposition}

Let us now study multiplicative properties of the charged fields $\psi_{s}$ ('operator product expansions'). For a generic charged operator $b \in \mathcal{B}$ one has the expansion formula (generalizing the harmonic analysis in the gauge symmetry case) implied by (4.4), (4.5)

$$
b=\lambda \mu\left(b V^{*}\right) V=\lambda \sum_{s} \mu\left(b \psi_{s}^{*}\right) \psi_{s} \quad(b \in \mathcal{B}) .
$$

In particular, by $(4.15)$,

$$
\psi_{t} \psi_{s}=\sum_{u} \lambda(e) T_{e} \psi_{u}
$$

where as before, $e$ is the channel $\varrho_{u} \prec \varrho_{t} \varrho_{s}$. We observe, that only charged fields with charge $\varrho_{u} \prec \varrho$ contribute to this operator product expansion, even if there are other sectors present in the DHR sector decomposition of $\varrho_{t} \varrho_{s}$. That this 'truncation of the fusion rules' is consistent, can be retraced, e.g., to the identity $(4.18(i i i))$ as follows.

Obviously, $\psi_{t} \psi_{s}$ is a charged intertwiner $: i d \rightarrow \varrho_{t} \varrho_{s}$, so one might expect that all charges $\varrho_{v}$ contained in $\varrho_{t} \varrho_{s}$ are interpolated by this composite field. But, in order to project a field carrying charge $\varrho_{v}$ out of $\psi_{t} \psi_{s}$, we have to multiply the latter with $T_{e}^{*}$ where $T_{e}: \varrho_{v} \rightarrow \varrho_{t} \varrho_{s}$. Now, computing $T_{e}^{*} \psi_{t} \psi_{s}$, or rather its image under $\varrho$, we get

$$
\varrho\left(T_{e}^{*} \psi_{t} \psi_{s}\right)=\varrho\left(T_{e}^{*} W_{t}^{*} V W_{s}^{*} V\right)=\varrho\left(T_{e}^{*} W_{t}^{*} \varrho\left(W_{s}^{*}\right) \cdot V V\right)=\varrho\left(T_{e}^{*} W_{t}^{*} \varrho\left(W_{s}^{*}\right)\right) \cdot X X
$$

Using $X X=\varrho(X) X$, we obtain an expression involving $\varrho\left[T_{e}^{*} W_{t}^{*} \varrho\left(W_{s}^{*}\right) X\right]$ where the argument in square brackets is an intertwiner $: \varrho \rightarrow \varrho_{v}$ in $\mathcal{A}$ which must vanish unless $\varrho_{v} \prec \varrho$. In other words, since the expansion (4.17) of $X$ contains only $T_{e}$ for fusion channels which are already contained in $\varrho$, it is annihilated by all $T_{e}$ leading to other channels. Therefore, the identity $T_{e}^{*} \psi_{t} \psi_{s}=0$ following from identity $(i i i)$ precisely describes in the operator product expansion for charged fields the suppression of channels $\varrho_{v}$ not contained in $\varrho$, i.e., the truncated fusion rules.

We now turn to our main result, the decomposition of correlation functions of charged fields into 'partial wave' contributions, and the decomposition of charged fields $\psi_{s}$ into 'chiral exchange fields'.

Applying the expansion (5.2) (and (4.11)) repeatedly, we find the following expansion for vacuum correlations of generic charged fields of the form $\varphi=\psi_{s}^{*} a$

$$
\left\langle\Omega, \varphi_{n} \cdots \varphi_{1} \Omega\right\rangle=\sum_{\xi} \prod_{i} \overline{\lambda\left(e_{i}\right)} \cdot\left\langle\Omega, T_{e_{n}}^{*} \varrho_{t_{n}}\left(a_{n}\right) \cdots T_{e_{2}}^{*} \varrho_{t_{2}}\left(a_{2}\right) T_{e_{1}}^{*} a_{1} \Omega\right\rangle
$$

where $T_{e_{i}}: \varrho_{u_{i}} \rightarrow \varrho_{t_{i}} \varrho_{s_{i}}$ and the sum extends over all vacuum-to-vacuum 'channels' of successive fusion $\xi=e_{n} \circ \cdots \circ e_{1}$ such that $t_{i}=u_{i-1}$ and $u_{n}=0=t_{1}$. The last step in this computation, the evaluation of a single charged field of the form $\psi_{s}^{*} a$ in the vacuum state, exploits the invariance of the vacuum state

$$
\omega\left(\psi_{s}^{*} a\right)=\omega\left(\mu\left(\psi_{s}\right)^{*} a\right)=\delta_{s 0} \lambda^{-1 / 2} \omega(a)
$$


since $\mu\left(\psi_{s}\right)=W^{*} \gamma\left(W_{s}^{*} V\right) W=W_{s}^{*} W^{*} X W=\delta_{s 0} \lambda^{-1 / 2} 1$. The factor $\lambda^{-1 / 2}$ is absorbed in the product in (5.3) in the guise of $\lambda\left(e_{1}\right)$ (note that for $\varrho_{t}=i d, T_{e}=1$, and $\tilde{T}_{e}=W W_{s} W_{s}^{*}$, one obtains $\left.\lambda(e)=W_{s}^{*} W^{*} X W_{s}=\lambda^{-1 / 2}\right)$.

In the formula (5.3), the single 'partial wave' contributions

$$
F_{\xi}=\left\langle\Omega, T_{e_{n}}^{*} \varrho_{t_{n}}\left(a_{n}\right) \cdots T_{e_{2}}^{*} \varrho_{t_{2}}\left(a_{2}\right) T_{e_{1}}^{*} a_{1} \Omega\right\rangle
$$

are kinematically distinguished correlation functions which depend only on the subtheory $\mathcal{A}$ and its superselection structure, but bear no reference to the field extension $\mathcal{B}$.

Proposition 5.1: The (local) $n$-point functions of charged fields from a field extension $\mathcal{B}$ have the partial wave expansions (5.3) where only the coefficients

$$
N_{\xi}=\prod_{i} \overline{\lambda\left(e_{i}\right)}
$$

involving the factors $\lambda\left(e_{i}\right)$ for every single transition in the channel of successive fusions, depend on $\mathcal{B}$.

On the Hilbert space of the representation $\left.\pi^{0}\right|_{\mathcal{A}} \equiv \bigoplus_{s} \pi_{s}$ (cf. Prop. 4.1) were also defined the 'reduced field bundle' operators $F(e, a)$ as a bounded operator version of chiral vertex operators $[6,7]$. If $e$ is the channel $\varrho_{u} \prec \varrho_{t} \varrho_{s}$, then $F(e, a) \equiv F(e, 1) \pi^{0}(a)$ interpolates from the subspace for $\pi_{t}$ to the subspace for $\pi_{u}$ by the formula

$$
F(e, a)|t ; \Psi\rangle:=\left|u ; \pi_{0}\left(T_{e}^{*} \varrho_{t}(a)\right) \Psi\right\rangle .
$$

These operators satisfy complicated 'exchange algebra' commutation relations (whence the name 'exchange fields' [30, 22]) involving matrix elements of the relevant statistics operators (braid matrices), and a multiplication law involving the fusion coefficients for the sectors. The algebra spanned by $F(e, a)$ is closed under multiplication and under the adjoint operation.

By inspection of the partial wave contributions (5.4) one sees that the latter are just the correlation functions of products of reduced field bundle operators $F(e, a)$. Therefore (5.3) implies the identification

$$
\psi_{s}^{*} a=\sum_{e} \overline{\lambda(e)} F(e, a)
$$

where the sum extends over all fusion channels with fixed charge label $s$. This formula is remarkable since the charged fields in $\mathcal{B}$ which satisfy local commutation relations and truncated fusion rules as discussed above, arise as specific linear combinations of reduced field bundle operators which satisfy exchange algebra commutation relations and do not exhibit truncation. Similarly, while every single partial wave contribution (5.4) is nonlocal, the sum (5.3) is a local $n$-point function. This is possible due to cancellations among the relevant fusion coefficients, which can be seen to follow from the system (4.18), (4.19) if written as a nonlinear system involving fusion coefficients and braid matrices along with the Clebsch-Gordan coefficients $\lambda(e)$. A similar statement applies to the identities

$$
\psi_{s}^{*}=d_{s}^{1 / 2} R_{s}^{*} \psi_{\bar{s}} \quad\left(R_{s}: i d \rightarrow \bar{\varrho}_{s} \varrho_{s} \text { isometric }\right)
$$

and

$$
\psi_{s}^{*} \psi_{s}=d_{s} / \lambda \cdot 1
$$

valid in $\mathcal{B}$, which we have not discussed here, but which can be proven within the reduced field bundle, with the identification (5.6), along the same lines. We refrain from working out the details here. 
Actually, the decomposition (5.6) can also be directly established in terms of the unitary equivalence between $\bigoplus \pi_{s}$ and $\left.\pi^{0}\right|_{\mathcal{A}}$.

It was argued in [7] that in a sufficiently regular theory with conformal covariance, scaling limits of $F(e, a)$ contracting the localization to a point $x$ should exist, and yield chiral vertex operators $\varphi_{e}(x)$ interpolating between the sectors $\mathcal{H}_{t}$ and $\mathcal{H}_{u}$ :

$$
\varphi_{e}(x) \sim \lim _{\lambda \rightarrow 0} \lambda^{-\Delta_{s}} F\left(e, \alpha_{x}^{(s)} \alpha_{\lambda}^{(s)}(a)\right)
$$

where $\alpha^{(s)}$ denotes the charge dependent effect of the translation $(x)$ resp. scale transformation $(\lambda)$ on the operator entry $a[7]$. It follows from these considerations that the pointlike limits of $\psi_{s}^{*} a$ yields local pointlike fields affiliated with $\mathcal{B}$ of the form

$$
\psi_{s}(x)=\sum_{e} \overline{\lambda(e)} \varphi_{e}(x)
$$

with the same coefficients as in (5.6). E.g., in the model (4.2) the heptuplett of primary currents $j^{a}(x)$ for the isospin 3 sector arise as linear combinations of vertex operators with coefficients $\lambda(e)$ to be computed below, and the same holds in general for charged local fields from $\mathcal{B}$.

In the pointlike limit, the partial wave contributions tend to 'conformal block functions'

$$
\mathcal{F}_{\xi}\left(x_{n}, \ldots, x_{1}\right)=\left\langle\Omega, \varphi_{e_{n}}\left(x_{n}\right) \cdots \varphi_{e_{1}}\left(x_{1}\right) \Omega\right\rangle
$$

to be identified with the $s$-channel blocks in the standard approach, determined from Ward identities and Knizhnik-Zamolodchikov equations [16]. For $n=4$, the label $\xi$ stands just for the intermediate sector due to $s$-channel fusion of the charges $s_{1}$ and $s_{2}$. Although the conformal blocks are non-local functions, their combinations with coefficients as in (5.3) are local $n$-point functions of local fields like $j^{a}(x)$.

The limit behaviour of partial waves (5.4) tending to conformal blocks (5.8) is an intrinsic property of the subtheory $\mathcal{A}$. As in Prop. 5.1, n-point functions of local charged fields $\psi_{s}(x)$ depend on the field extension $\mathcal{B}$ only through the expansion coefficients $N_{\xi}$ given by $(5.5)$.

In practice, we don't know the absolute normalizations of the limiting functions (5.8) in order to identify them with a choice of $s$-channel solutions as in Sects. 2 and 3, nor do we know the relative normalizations of different partial wave contributions with respect to each other. This would require the control of the previously mentioned pointlike limits which is in general a difficult problem. However, one can compute double ratios, which compare two different field extensions, of the form

$$
\frac{N_{\xi}^{\prime} / N_{\eta}^{\prime}}{N_{\xi} / N_{\eta}}
$$

which are completely normalization independent 'characteristic' quantities. These double ratios must in particular coincide with the corresponding double ratios comparing relative amplitudes of $s$-channel conformal block functions contributing to $n$-point functions of point-like fields from two different field extensions, as computed in Sects. 2 and 3.

Since the double ratios (5.9) are given by (5.5), we have established the desired relation between relative amplitudes of conformal blocks and the data of the relevant local subfactors. This relation is based on the identification of the expansion coefficients in (4.17) for the characteristic isometry and in (5.2) for operator products of charged fields (reflected also in (5.6) for charged fields as elements of the reduced field bundle). 
Let us now compute the amplitudes (5.5) for our first model (4.2) from the characteristic triple $(\varrho, W, X)$. From the branching of the vacuum representation of $\mathcal{B}$ upon restriction to $\mathcal{A}$, we know that $\varrho \simeq \varrho_{0} \oplus \varrho_{3}$ (see Sect. 4). By (4.10), the index is $\lambda=d(\varrho)=d_{0}+d_{3}=$ $3+\sqrt{3}$. Actually, finite index type $I I I_{1}$ subfactors are isomorphic to type $I I_{1}$ subfactors tensored with a type $I I I$ factor [31]. The corresponding type $I I_{1}$ subfactor associated with the model (4.2) is the well known subfactor of index $\lambda=3+\sqrt{3}$ constructed in [32].

Choosing $\varrho_{0}=i d$ in its equivalence class, the isometry $W: i d \rightarrow \varrho$ is uniquely determined up to an irrelevant phase. The coefficients $\lambda(e)$ for the isometry $X$ can be computed from $X^{*} X=1 l$ and the identity $(4.18(i))$ : there are only five fusion channels $\varrho_{u} \prec \varrho_{t} \varrho_{s}$ with all $\varrho_{s}, \varrho_{t}, \varrho_{u} \prec \varrho$, with which we associate isometric intertwiners as follows:

$$
T_{a}: \varrho_{0} \rightarrow \varrho_{0} \varrho_{0} \quad T_{b}: \varrho_{3} \rightarrow \varrho_{3} \varrho_{0} \quad T_{c}: \varrho_{3} \rightarrow \varrho_{0} \varrho_{3} \quad T_{d}: \varrho_{0} \rightarrow \varrho_{3} \varrho_{3} \quad T_{e}: \varrho_{3} \rightarrow \varrho_{3} \varrho_{3}
$$

Since $\varrho_{0}=i d$, we may choose $T_{a}=T_{b}=T_{c}=1$. According to standard notation $[5,6,7]$, we call $R$ the isometry $T_{d}: i d \rightarrow \varrho_{3}^{2}$. We have therefore:

$$
\begin{aligned}
& X=\lambda(a) \cdot \varrho\left(W_{0}\right) W_{0} W_{0}^{*}+\lambda(b) \cdot \varrho\left(W_{0}\right) W_{3} W_{3}^{*}+ \\
& \quad+\lambda(c) \cdot \varrho\left(W_{3}\right) W_{0} W_{3}^{*}+\lambda(d) \cdot \varrho\left(W_{3}\right) W_{3} R W_{0}^{*}+\lambda(e) \cdot \varrho\left(W_{3}\right) W_{3} T_{e} W_{3}^{*},
\end{aligned}
$$

where $W_{0} \equiv W: i d \rightarrow \varrho$ and $W_{3}: \varrho_{3} \rightarrow \varrho$ are orthonormal isometries, and $E_{0}=W_{0} W_{0}^{*}$ and $E_{3}=W_{3} W_{3}^{*}$ are complementary projections in the commutant of $\varrho$ onto the two subsectors of $\varrho$. Then $(4.18(i))$ reads

$$
\begin{gathered}
W_{0}^{*} X=\lambda(a) E_{0}+\lambda(c) E_{3}=\lambda^{-1 / 2} 1 \\
\varrho\left(W_{0}^{*}\right) X=\lambda(a) E_{0}+\lambda(b) E_{3}=\lambda^{-1 / 2} 1
\end{gathered}
$$

hence $\lambda(a)=\lambda(b)=\lambda(c)=\lambda^{-1 / 2}$. We are free to choose the complex phases of $R$ and $T_{e}$ such that $\lambda(d)$ and $\lambda(e)$ are also positive. Now, the isometricity of $X$ together with the orthogonality of $R$ and $T_{e}$ (i.e., $R^{*} T_{e}=0$ ) implies

$$
X^{*} X=\left[\lambda(a)^{2}+\lambda(d)^{2}\right] E_{0}+\left[\lambda(b)^{2}+\lambda(c)^{2}+\lambda(e)^{2}\right] E_{3}=1 l
$$

hence $\lambda(d)=\sqrt{1-\lambda^{-1}}$ and $\lambda(e)=\sqrt{1-2 \lambda^{-1}}$. We don't need to verify the remaining identities (4.18), (4.19) since we know that the extension is local and yields a subfactor of index $\lambda=3+\sqrt{3}$. (Unfortunately, the computation is much less trivial for the other, $E_{8}$, extension treated in Sects. 2 and 3.)

For charged fields with $\varrho_{s}=\varrho_{3}$, only the channels $c \equiv(30), d \equiv(03), e \equiv(33)$ are relevant $\left((J I)\right.$ stands for an exchange field of charge 3 acting on $\mathcal{H}_{I}$ with values in $\mathcal{H}_{J}$.) Therefore, we have

$$
\lambda(30)=\lambda^{-1 / 2}, \quad \lambda(03)=\left(\frac{\lambda-1}{\lambda}\right)^{1 / 2}, \quad \lambda(33)=\left(\frac{\lambda-2}{\lambda}\right)^{1 / 2} .
$$

This gives for the ratio of the amplitudes of the conformal blocks with intermediate $s$ channel $I=0,3$ contributing to the 4-point function of the isospin 3 field

$$
N_{3} / N_{0}=\frac{\lambda(03) \lambda(33) \lambda(33) \lambda(30)}{\lambda(03) \lambda(30) \lambda(03) \lambda(30)}=\frac{\lambda-2}{\sqrt{\lambda-1}}=\sqrt{2} .
$$

As discussed before, due to uncontrolled normalizations, one has to compute double ratios like (5.9) of relative amplitudes comparing two different field extensions. Indeed, there is always a 'standard' extension to compare with, which specializes for chiral current algebras to the $A$ series of modular invariants [3], and therefore yield the diagonal extensions as in our model (4.3). 
Proposition 5.2: [33, 9] For rational chiral theories $\mathcal{A}_{c h}$ (i.e., theories with only a finite number of superselection sectors $\pi_{s}$ with finite statistics), $\varrho \simeq$ $\bigoplus_{s} \varrho_{s} \otimes \bar{\varrho}_{s}$ is a canonical endomorphism of $\mathcal{A}^{(2)} \equiv \mathcal{A}_{c h} \otimes \mathcal{A}_{c h}$, giving rise to a local two-dimensional field extension $\mathcal{B}^{(2)}$.

This result is a corollary to the computation in [33] of the associated characteristic isometry $X^{(2)}$ satisfying the system of identities (4.18), (4.19). The vacuum representation of this extension contains all 'diagonal' sectors of $\mathcal{A}^{(2)}$ of the form $\pi_{s} \otimes \pi_{\bar{s}}$ precisely once.

It is more convenient to deviate from the basis conventions in [33] and choose a $C P T$ conjugate pair of bases of isometric intertwiners $T_{e}$ and $T_{\bar{e}}=j\left(T_{e}\right)$ on the two chiral lightcones (cf. [9]). The anti-linear CPT conjugation $j$ is an appropriate Tomita-Takesaki modular conjugation [24,34]. It acts like a reflection $x \leftrightarrow-x$ on the algebras of chiral intervals, and relates conjugate sectors $\varrho \leftrightarrow \varrho=j \circ \varrho \circ j$. In such a basis, the isometry $X^{(2)}$ is simply

$$
X^{(2)}=\Lambda^{-1 / 2} \sum_{e} \sqrt{\frac{d_{t} d_{s}}{d_{u}}} \tilde{T}_{e} \otimes \tilde{T}_{\bar{e}}
$$

where $\tilde{T}_{e}$ are local intertwiners in $\mathcal{A}_{c h}$ as in (4.17) corresponding to the fusion channels $\varrho_{u} \prec \varrho_{t} \circ \varrho_{s}$ as before, $\tilde{T}_{\bar{e}}=j\left(\tilde{T}_{e}\right)$ correspond to the $C P T$ conjugate channel $\bar{\varrho}_{u} \prec \bar{\varrho}_{t} \bar{\varrho}_{s}$, and $d_{s}$ are the statistical dimensions of $\varrho_{s}$. The index equals $\Lambda=\sum_{s} d_{s}^{2}$. The fusion channels contributing to the isometry $X^{(2)}$ for the two-dimensional subtheory (4.3) are of the form $e \otimes \bar{e}$, and the coefficients $\lambda^{(2)}(e \otimes \bar{e})$ are read off eq. (5.11). The fact that the corresponding two-dimensional fields

$$
\left.\Phi_{s}=\sum_{e \otimes \bar{e}} \sqrt{\frac{d_{t} d_{s}}{d_{u}}} F(e \otimes \bar{e}, 1] \otimes \mathbb{l}\right) \equiv \sum_{e} \sqrt{\frac{d_{t} d_{s}}{d_{u}}} F(e, 1) \otimes F(\bar{e}, 1)
$$

contracted from chiral exchange fields of fixed charge $[s],[\bar{s}]$ are indeed local fields acting on the Hilbert space $\mathcal{H}^{(2)}=\bigoplus_{t} \mathcal{H}_{t} \otimes \mathcal{H}_{\bar{t}}$, was established in [22]. Although the diagonal sectors are not closed under composition whenever there are non-simple fusion rules among the chiral sectors $\pi_{s}$, the operator product of the diagonal fields $\Phi_{s}$ contains only other diagonal fields due to cancellations among the fusion coefficients. This is another instance of truncated fusion rules.

From

$$
\lambda^{(2)}(e \otimes \bar{e})=\sqrt{\frac{d_{s}}{\Lambda}} \sqrt{\frac{d_{t}}{d_{u}}}
$$

it is obvious that the amplitudes for the $2 \mathrm{D}$ partial waves contributing to a given $n$-point function of integer isospin fields $\left\langle\Omega, \Phi_{n} \cdots \Phi_{1} \Omega\right\rangle=\sum_{\xi} N_{\xi \otimes \bar{\xi}}^{(2)} \mathcal{F}_{\xi} \cdot \overline{\mathcal{F}}_{\bar{\xi}}$ are all equal:

$$
N_{\xi \otimes \bar{\xi}}^{(2)}=\prod_{i} \sqrt{d_{s_{i}} / \Lambda} \propto 1
$$

Given the diagonal standard extension, we can predict characteristic invariants for every other extension which can be read off the respective $n$-point functions, independent of all normalizations of partial waves and conformal blocks, by taking double ratios of amplitudes (5.5) and (5.12)

$$
\frac{\left(N_{\xi} / N_{\eta}\right)\left(N_{\bar{\xi}} / N_{\bar{\eta}}\right)}{N_{\xi \otimes \bar{\xi}}^{(2)} / N_{\eta \otimes \bar{\eta}}^{(2)}}=\prod_{i} \frac{\lambda\left(e_{i}\right) \bar{\lambda}\left(\bar{e}_{i}\right)}{\lambda\left(f_{i}\right) \bar{\lambda}\left(\bar{f}_{i}\right)}=\prod_{i} \frac{\left|\lambda\left(e_{i}\right)\right|^{2}}{\left|\lambda\left(f_{i}\right)\right|^{2}} .
$$

Here we have used the fact that the coefficients of $X$ and $j(X)$ in $C P T$ conjugate bases are complex conjugates, $\bar{\lambda}(\bar{e})=\overline{\lambda(e)}$. E.g., for the 4-point function of the isospin 3 field 
in the $E_{6}$ model (4.2), we get

$$
\frac{\left(N_{3} / N_{0}\right)^{2}}{N_{3}^{(2)} / N_{0}^{(2)}}=2
$$

in agreement with the result obtained previously (eq. (3.8) and [12]) by the analysis of locality in terms of explicit conformal block functions given as solutions to KZ differential equations.

We emphasize that this method works for every 'non-diagonal' extension of a given chiral theory without controlling the actual pointlike limits $F(e, a) \rightarrow \varphi_{e}(x)$, since there is always the 'diagonal' one to compare with. Moreover, it immediately applies to mixed and higher $n$-point functions.

We conclude this section with another instructive (albeit almost trivial) example giving rise to anyonic field extensions. We consider a local theory $\mathcal{A}$ with $N$ simple superselection sectors $\varrho_{s}$ with $\mathbb{Z}_{N}$ fusion rules $[s][t]=[s+t(\bmod N)]$. For simplicity, assume that the automorphisms $\varrho_{s}$ can be chosen to satisfy $\varrho_{s} \varrho_{t}=\varrho_{s+t}($ understood $\bmod N)$, by which all intertwiners $T_{e}$ of the general analysis are trivial $=1 l$. This choice is always possible for odd $N$, and for even $N$ provided the fractional spin of $\varrho_{s}$ satisfies $N \Delta_{s} \in \mathbb{Z}$ (cf. [22]). The sector structure is that of the simple sectors in $s u(N)$ current algebras. It also occurs in the models constructed in [35], where, however, the violation of the spin condition leads to a minor complication which we want to ignore here. The case $N=2$ includes the $D_{n}$ series of chiral $s u(2)$ current algebra extensions.

We choose a complete system of orthonormal isometries $W_{s}$ and construct the reducible endomorphism $\varrho(a):=\sum_{s} W_{s} \varrho_{s}(a) W_{s}^{*}$. Then the triple $(\varrho, W, X)$ where $W=W_{0}$ and

$$
X:=N^{-1 / 2} \sum_{s t} \varrho\left(W_{s}\right) W_{t} W_{s+t}^{*}
$$

(with trivial Clebsch-Gordan coefficients for an abelian group) solves the system (4.18). The charged fields $\psi_{s}$ are obtained (up to a normalization factor $N^{1 / 2}$ ) as the unitary shift operators $|t ; \Psi\rangle \mapsto|t+s ; \Psi\rangle$ on $\bigoplus_{t} \mathcal{H}_{t}$. They satisfy $\psi_{s} \psi_{t}=\psi_{s+t}$ and implement the endomorphisms $\varrho_{s}$ (in the representation $\pi^{0}=\bigoplus \pi_{s}$ )

$$
\varrho_{s}(a)=\psi_{s} a \psi_{s}^{*} \quad(a \in \mathcal{A}) .
$$

The gauge group $\mathbb{Z}_{N}$ acts by $\gamma_{n}\left(\psi_{s}\right)=e^{2 \pi i n s / N} \psi_{s}$ with average $\mu\left(\psi_{s}\right)=\delta_{s 0}$ ll. Putting

$$
V:=N^{-1 / 2} \sum_{s} W_{s} \psi_{s}
$$

and defining $\gamma$ by (4.6) with index $\lambda=\left|\mathbb{Z}_{N}\right|=N$, then $\gamma(V)=X$ and the triple $(\gamma, V, W)$ satisfies the identities (4.4). Adjoining the charged fields $\psi_{s}$ to the local algebras, we obtain an anyonic field extension $\mathcal{B}$ by the simple sectors of $\mathcal{A}$.

\section{Concluding remarks}

The old hope that the 'germ of the observable algebra' generated by the internal symmetry currents and the stress-energy tensor completely determines a local quantum field theory turns out to require some qualifications. Two-dimensional conformal current algebra models tell us that depending on the value of the level $k$ (which characterizes both the algebra $\mathcal{A}_{k}$ and the vacuum state of the theory), there may be several - one, two,

or three for $\mathcal{A}_{k}(s u(2))$ - local conformal field theories corresponding to the same vacuum representation of $\mathcal{A}_{k}$. 
The different theories are distinguished by different maximal local chiral extensions $\mathcal{B}_{k}$ and by different braid invariant quadratic forms $M$. The primary local chiral fields which extend $\mathcal{A}_{k}$ obey fusion rules which are majorized by the intrinsic DHR fusion rules of superselection sectors. Both the invariant ratios of structure constants which are characteristic quantities for local field extensions, and the truncated fusion rules are understood and computed in conventional field theoretical terms and in terms of the theory of subfactors applied to a single local subfactor $\mathcal{A}(\mathcal{J}) \subset \mathcal{B}(\mathcal{J})$.

Our field theoretical computation uses a closed expression for the $s$-channel fusion matrix (that is already implicit in [13]) which has the virtue of displaying their invariance under Galois automorphisms (the individual structure constants as well as the matrix elements of the monodromy representation of the mapping class group belonging to the same algebraic number field). The relevance of such arithmetic properties has been recently exhibited in a study of the Schwarz problem ('When is the representation of the braid group a finite matrix group?') for the KZ equation [20].

On the other hand, the application of the theory of finite index subfactors to local field extensions gives a natural interpretation of the field theoretical structures in terms of a generalized 'harmonic analysis'. The 'irreducible tensor operators' of this analysis are the quantum field theoretical charged intertwiners. This approach is very close to the spirit of Ocneanu who first considered subfactors as 'generalized groups', but gives more evidence to this view than the combinatorial description in terms of bi-partite graphs and connections [36]. Part of Ocneanu's induction-restriction graph is reflected in the 'truncated fusion rules' which in turn derive from harmonic analysis in the form of operator product expansions for charged fields. Through Longo's theorem relating the truncation to the depth of the inclusion [28], it is nicely exhibited that the generalized symmetry associated with conformal embeddings is not given by a Hopf $C^{*}$ algebra in general. Longo's characterization of a subfactor in terms of a triple $(\varrho, W, X)$ gives rise to a notion of generalized Clebsch-Gordan coefficients which does not refer to any assumed linear transformation law of the irreducible tensor operators. We note that the interpretation of these structures as a generalized symmetry is not imposed but emerges naturally from the theory of subfactors.

When one compares our two different approaches, one can also observe some unbalance. E.g., the role of the Galois automorphisms is not yet understood in terms of the subfactor approach. In particular, the Galois group acting on the structure constants does not map a unitary theory into another unitary theory, nor are there any 'Galois relatives' of a subfactor. Indeed, the characteristic ratios of structure constants like (3.8), (3.9), (3.15) resp. (5.13) turn out to be rational numbers and are, therefore, Galois invariants.

The characterization of a local extension in terms of a triple $(\varrho, W, X)$ as in Prop. 4.2 logically proceeds in two steps: first, one has to solve the system (4.18) which, among other things, controls the consistent truncated operator product expansions. This already yields field extensions which, however, may be non-local. E.g., a fermionic field theory as an extension of its even (bosonic) subtheory arises in this way. The locality condition (4.19) is only imposed in a second step. On the other hand, in the conformal block approach the locality condition seems to be the only vital step. In fact, we consider the analogue of the first step to be hidden in the KZ equation, whose solutions automatically give rise to a consistent fusion.

Acknowledgements: The discussions that gave rise to the present paper started when all three authors were visiting the Erwin Schrödinger International Institute for Mathematical Physics in Vienna in early 1993. Most of the work was done during a second visit at 
the ESI by Ya.S. and I.T. in the spring of 1994. We all benefited from the hospitality of the collegues in Vienna and of the creative atmosphere at the Schrödinger Institute. I.T. thanks J.-B. Zuber for a stimulating discussion on the special properties of ratios of structure constants, and for acquainting him with the work in progress of V. Petkova and himself [37] that relates these constants to the eigenvectors of associated Cartan matrices. Ya.S. and I.T. acknowledge partial support by the Bulgarian Foundation for Scientific Research under contract F-11.

\section{References}

[1] V.F.R. Jones: Index for subfactors, Invent. Math. 72, 1-25 (1983);

Braid groups, Hecke algebras, and type $I I_{1}$ factors, in: Geometric Methods in Operator Algebras (Proc. of US-Japan Seminar, Kyoto 1983), Pitman Research Notes in Math. 123, 242-273 (1986).

[2] V. Pasquier: Operator content of the ADE lattice models, J. Phys. A: Math. Gen. 20, 5707-5717 (1987).

[3] A. Cappelli, C. Itzykson, J.-B. Zuber: The ADE classification of minimal and $A_{1}^{(1)}$ conformal invariant theories, Commun. Math. Phys. 113, 1-26 (1987).

[4] R. Haag, D. Kastler: An algebraic approach to quantum field theory, Journ. Math. Phys. 5, 848-861 (1964).

[5] S. Doplicher, R. Haag, J.E. Roberts: Local observables and particle statistics. I+II, Commun. Math. Phys. 23, 199-230 (1971) and 35, 49-85 (1974);

R. Haag: Local Quantum Physics, Texts and Monographs in Physics, Springer 1992.

[6] K. Fredenhagen, K.-H. Rehren, B. Schroer: Superselection sectors with braid group statistics and exchange algebras. I, Commun. Math. Phys. 125, 201-226 (1989).

[7] K. Fredenhagen, K.-H. Rehren, B. Schroer: Superselection sectors with braid group statistics and exchange algebras. II, Rev. Math. Phys. Special issue, 113-157 (1992).

[8] R. Longo: Index of subfactors and statistics of quantum fields. I+II, Commun. Math. Phys. 126, 217-247 (1989) and Commun. Math. Phys. 130, 285-309 (1990).

[9] R. Longo, K.-H. Rehren: Nets of subfactors, in preparation, based on joint work with K. Fredenhagen and J.E. Roberts; for the general ideas see:

K.-H. Rehren: Subfactors and coset models, in: Generalized Symmetries in Physics, V. Dobrev et al. eds., (World Scientific, Singapore 1994), pp. 338-356.

[10] A. Schellekens, N. Warner: Conformal subalgebras of Kac-Moody algebras, Phys. Rev. D34, 3092-3101 (1986);

P. Bouwknegt, W. Nahm: Realization of the exceptional modular invariant $A_{1}^{(1)}$ partition functions, Phys. Lett. B184, 359-362 (1987);

F.A. Bais, P.G. Bouwknegt: A classification of subgroup truncations of the bosonic string, Nucl. Phys. B279, 561-570 (1987).

[11] E. Witten, Non-Abelian bosonization in two dimensions, Commun. Math. Phys. 92, 445-472 (1984).

[12] J. Fuchs, A. Klemm: The computation of the operator algebra in non-diagonal conformal field theories, Ann. Phys. (N.Y.) 194, 303 (1989);

J. Fuchs, A. Klemm, C. Scheich: The operator algebra of the $E_{8}$ type $S U_{2} W Z W$ theory, Zeitschr. f. Phys. C46, 71-74 (1990);

P. Furlan, A.Ch. Ganchev, V.B. Petkova: Fusion matrices and $c<1$ (quasi) local conformal theories, Int. J. Mod. Phys. A5, 2721 (1990).

[13] Ya.S. Stanev, I.T. Todorov, L.K. Hadjiivanov: Braid invariant rational conformal models with a quantum group symmetry, Phys. Lett. B276, 87-94 (1992).

[14] Ya.S. Stanev, I.T. Todorov, L.K. Hadjiivanov: Braid invariant chiral conformal models with a quantum group symmetry, in: Quantum Symmetries, H.-D. Doebner, V.K. Dobrev eds., (World Scientific, Singapore 1993), pp. 24-40. 
[15] A. Tsuchiya, Y. Kanie: Vertex operators in conformal field theory on $\mathbb{P}^{1}$ and monodromy representations of braid group, in: Conformal Field Theory and Solvable Lattice Models, Adv. Stud. Pure Math. 16, 297-372 (1988); and II: Lett. Math. Phys. 13, 303-312 (1987).

[16] V.G. Knizhnik, A.B. Zamolodchikov: Current algebra and Wess-Zumino model in two dimensions, Nucl. Phys. B247, 83-103 (1984).

[17] S. Doplicher, J.E. Roberts: Why there is a field algebra with a compact gauge group describing the superselection structure in particle physics, Commun. Math. Phys. 131, 51-107 (1990).

[18] G. Felder, K. Gawȩdzki, A. Kupiainen: The spectrum of Wess-Zumino-Witten models, Nucl. Phys. B299, 355-366 (1988).

[19] I.T. Todorov: What are we learning from 2-dimensional conformal models, lecture presented at the Workshop on Mathematical Physics 'Towards the $21^{\text {st }}$ Century', Beer Sheva, Israel, Vienna preprint ESI 52 (1993).

[20] Ya.S. Stanev, I.T. Todorov: On Schwarz problem for the $\widehat{s u}_{2}$ Knizhnik-Zamolodchikov equation, Vienna preprint ESI 121 (1994), submitted to Lett. Math. Phys.

[21] L. Michel, Ya.S. Stanev, I.T. Todorov: D-E classification of the local extensions of $s_{2}$ current algebras, Teor. Mat. Fiz. 92, 507-521 (1992) (Theor. Math. Phys. 92, 1063 (1993)).

[22] K.-H. Rehren: Space-time fields and exchange fields, Commun. Math. Phys. 132, 461-483 (1990).

[23] D. Buchholz, C. D'Antoni, K. Fredenhagen: The universal structure of local algebras, Commun. Math. Phys. 111, 123-135 (1987).

[24] F. Gabbiani, J. Fröhlich: Operator algebras and conformal field theory, Commun. Math. Phys. 155, 569-640 (1993).

[25] H. Kosaki: Extension of Jones' theory on index to arbitrary factors, J. Funct. Anal. 66, 123-140 (1986).

[26] M. Pimsner, S. Popa: Entropy and index for subfactors, Ann. Sci. Éc. Norm. Sup. 19, 57-106 (1986).

[27] A. Wassermann: Subfactors arising from positive energy representations of some infinite dimensional groups, unpublished notes 1990.

[28] R. Longo: A duality for Hopf algebras and for subfactors. I, Commun. Math. Phys. 159, 133-150 (1994).

[29] G. Moore, N. Seiberg: Polynomial equations for rational conformal field theories, Phys. Lett. 212B, 451-460 (1988).

[30] K.-H. Rehren, B. Schroer: Einstein causality and Artin braids, Nucl. Phys. B312, 715-750 (1989).

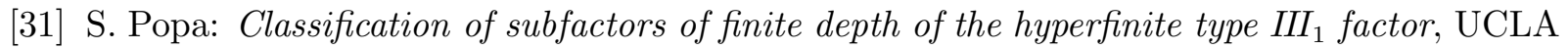
preprint (1994); KHR is indebted to A. Wassermann who informed him of this result.

[32] F. Goodman, P. de la Harpe, V. Jones: Coxeter graphs and towers of algebras, MSRI Publ. 14 (Springer 1989), Chap. 4.5.

[33] K.-H. Rehren: Field operators for anyons and plektons, Commun. Math. Phys. 145, 123-148 (1992); cf. in particular Prop. 2.6. and the 'Notes added in proof'.

[34] R. Brunetti, D. Guido, R. Longo: Modular structure and duality in conformal quantum field theory, Commun. Math. Phys. 156, 201-219 (1993);

H.-W. Wiesbrock: Conformal quantum field theory and half-sided modular inclusions of von Neumann algebras, Commun. Math. Phys. 158, 537-544 (1993).

[35] D. Buchholz, G. Mack, I. Todorov: The current algebra on the circle as a germ for local field theories, Nucl. Phys. B (Proc.Suppl.) 5B, 20-56 (1988).

[36] A. Ocneanu: Quantized groups, string algebras, and Galois theory for algebras, in: Operator Algebras and Applications, Vol. 2, eds. D.E. Evans et al., London Math. Soc. Lecture Notes Series 135, p. 119 (Cambridge 1988).

[37] V.B. Petkova, J.-B. Zuber: On structure constants of sl(2) conformal theories, Clausthal/Saclay preprint (to appear). 